THESIS

MOTIVATIONS FOR PARTICIPATION IN KNITTING AMONG YOUNG WOMEN

\author{
Submitted by \\ Casey Rhea Stannard \\ Department of Design and Merchandising
}

In partial fulfillment of the requirements

For the Degree of Master of Science

Colorado State University

Fort Collins, Colorado

Summer 2011

Master’s Committee:

Advisor: Eulanda Sanders

Ajoy Sarkar

Suzanne Faris 
Copyright by Casey Rhea Stannard 2011

All Rights Reserved 


\section{ABSTRACT MOTIVATIONS FOR PARTICIPATION IN KNITTING AMONG YOUNG WOMEN}

The purpose of this research was to understand the complex motivations of young women who choose to participate in knitting. The number of young women who knit has increased dramatically in the past ten years. However, there has been little research focused on understanding the culture of these young, female knitters and no research has concentrated its attention on the material culture of these women and the artifacts they produce.

Fifteen young, female knitters (ages 18-30) who resided in Northern Colorado were identified to participate in the study. Data were collected from in-depth interviews, a material culture analysis of artifact(s) made by the participants, and a demographic data survey. Interviews were recorded, transcribed, and artifacts made by the participants were photographed for later analysis. Validity was established through the use of multiple information sources and a secondary coder. Data gathered from the interviews were analyzed using constant comparative method. The knitted artifacts were analyzed using material culture and content analysis methods. Finally, the survey data were examined using frequencies and descriptive statistics.

Thirteen subthemes emerged during the coding process of the interview data: creativity, multi-tasking, financial, alterations, information sources, social aspects, negative reactions, mistakes, expense, positive reactions, products, confidence, and 
relaxation. These themes were grouped into three main categories: incentives, barriers, and positive outcomes. The Taxonomy of Participation in Knitting was developed to graphically arrange the themes and subthemes.

The data were then compared to an existing theory, Uses and Gratifications (UG), to determine if the categories of motivations described by Katz et al. (1974) were appropriate to describe the incentives for young women to participate in knitting. The categories of UG were very suitable and a second model, The Model of Motivations for Knitting among Young Women, was created to showcase the motivations that participants had for engaging in knitting.

The research is significant to young knitters as it serves as one of the only attempts to understand and describe their culture. It is also worthwhile to retailers involved in knitting related pursuits as it uncovers preferences for yarns, patterns, and stitches among young, female knitters. Finally, the research is valuable to the academic community as it helps to understand these knitters and created a study to reference as well as models to be utilized. 


\section{TABLE OF CONTENTS}

Chapter One: Introduction

Background

Purpose

Research Questions

Objectives

Assumptions

Scope and Limitations

Definitions

$\begin{array}{lr}\text { Chapter Two: Review of Literature } & 10\end{array}$

$\begin{array}{ll}\text { Introduction } & 10\end{array}$

History of Knitting 11

Early Knitting 11

Framework Knitting $\quad 11$

Knitting in America $\quad 12$

Victorian Fancy Knits 13

Knitting For the Troops 14

The Roaring Twenties and Knitting $\quad 15$

Knitting Through the Forties and Fifties $\quad 15$

Feminism Calls for the End of Knitting 16

September 11, 2001 and the Resurgence of Knitting 17

$\begin{array}{lr}\text { Personal Benefits of Knitting } & 18\end{array}$

$\begin{array}{lr}\text { Psychological } & 18\end{array}$

$\begin{array}{lr}\text { Physiological } & 19\end{array}$

Meaning of Knitted Artifacts 19

$\begin{array}{ll}\text { Creativity through Knitting } & 19\end{array}$

Knitted Gifts 20

Social benefits of Knitting Circles $\quad 21$

$\begin{array}{ll}\text { Camaraderie } & 21 \\ & 21\end{array}$

$\begin{array}{ll}\text { Social Support } & 21\end{array}$

$\begin{array}{ll}\text { Social Networking } & 22\end{array}$

Visibility of Knitters 23

The Celebrity of Knitting 23

$\begin{array}{ll}\text { Knitivism } & 24\end{array}$

The Craft Marketplace $\quad 26$

Knitting Supply Retailers $\quad 26$

$\begin{array}{ll}\text { Materials } & 27\end{array}$

$\begin{array}{ll}\text { Knitting Publications } & 29\end{array}$

$\begin{array}{ll}\text { Theoretical Frameworks } & 29\end{array}$

Uses and Gratifications $\quad 30$

Application to Present Study 32 
$\begin{array}{ll}\text { Material Culture } & 34\end{array}$

Application to Present Study 36

Relationships of Theoretical Frameworks 36

Critical Evaluation of Existing Work 36

Chapter Three: Methods and Procedures 38

Introduction 38

Pilot Testing 39

Sample Selection $\quad 39$

$\begin{array}{ll}\text { Data Collection } & 40\end{array}$

Interviews $\quad 40$

Knitted Artifacts $\quad 40$

Post Interview Survey $\quad 42$

Data Analysis $\quad 43$

Interviews 43

Knitted Artifacts $\quad 44$

Post Interview Survey $\quad 45$

Validity and Reliability

$\begin{array}{ll}\text { Validity } & 45\end{array}$

$\begin{array}{ll}\text { Reliability } & 46\end{array}$

Chapter Four: Results 48

$\begin{array}{ll}\text { Participant Demographics } & 48\end{array}$

Interview Data 53

Incentives $\quad 54$

Creativity $\quad 55$

Multi-tasking $\quad 56$

Financial $\quad 57$

$\begin{array}{ll}\text { Alterations } & 58\end{array}$

Information Sources $\quad 59$

Social Aspects 61

Barriers $\quad 63$

Negative Reactions $\quad 64$

$\begin{array}{ll}\text { Mistakes } & 65\end{array}$

Expense $\quad 67$

$\begin{array}{ll}\text { Outcomes } & 67\end{array}$

Positive Reactions $\quad 68$

$\begin{array}{ll}\text { Products } & 69\end{array}$

$\begin{array}{ll}\text { Confidence } & 71\end{array}$

Relaxation $\quad 72$

Knitted Artifacts

Materials $\quad 73$

$\begin{array}{ll}\text { Construction } & 76\end{array}$

$\begin{array}{ll}\text { Provenance } & 77\end{array}$

$\begin{array}{ll}\text { Function } & 79\end{array}$

$\begin{array}{ll}\text { Value } & 82\end{array}$ 
Chapter Five: Discussion $\quad 85$

Introduction $\quad 85$

Interview Data $\quad 85$

$\begin{array}{ll}\text { Knitted Artifacts } & 88\end{array}$

Participant Demographics $\quad 90$

Research Questions $\quad 92$

Research Question Number One $\quad 92$

$\begin{array}{ll}\text { Motivations } & 92\end{array}$

Benefits $\quad 94$

$\begin{array}{ll}\text { Meanings } & 94\end{array}$

Application Theory $\quad 96$

Research Question Number Two 99

Research Question Number Three 101

$\begin{array}{ll}\text { Research Question Number Four } & 103\end{array}$

$\begin{array}{ll}\text { Social Culture } & 104\end{array}$

Political Culture 105

$\begin{array}{ll}\text { Model } & 107\end{array}$

Chapter Six: Conclusion $\quad 110$

Significance 111

Implications 112

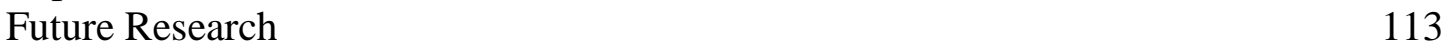

$\begin{array}{ll}\text { References } & 115\end{array}$

$\begin{array}{ll}\text { Appendix A } & 121\end{array}$

Examples of Knitivism

$\begin{array}{ll}\text { Appendix B } & 122\end{array}$

Interview Schedule

$\begin{array}{ll}\text { Appendix C } & 123\end{array}$

Knitted Artifact Instrument
Appendix D

Participant for Research Flyer

$\begin{array}{ll}\text { Appendix E } & 125\end{array}$

Post Interview Participant Survey 


\section{CHAPTER ONE}

\section{INTRODUCTION}

\section{Background}

Knitting has undergone a significant and somewhat surprising revival in the new millennium. Once viewed as a domestic chore practiced by "grannies," knitters are shedding this stereotype and are knitting on New York City subways, in crowded pubs, and other trendy places. Celebrities, young urban professionals, feminists, and even men are participating in the traditional craft. By examining a few statistics on knitting the explosive growth in popularity and participation becomes even more apparent. For example, Myzelev (2009) indicated that the number of knitting books published (465 total) from 2000-2007 was almost double the amount published from 1980-1990. Similarly, the number of women under 45 who were knowledgeable about knitting increased from nine to eighteen percent from 1996 to 2002 (Minahan \& Wolfram Cox, 2007).

The growth of knitting can be attributed to societal forces such as: the appropriation of craft as a medium for expression by the fine art community, changes in leisure, interest in social and political activism, as well as consumption of sustainable materials. Abrams (2006) proclaimed, "today hand-knitting is regarded as a creative and 
skilled endeavor which is undergoing a revival and a re-evaluation by means of the reappropriation and adaptation of traditional skills by modern artists and designers” (p. 150). Many fine artists have adopted knitting to convey messages about the relationship between art and craft as well as femininity and feminism (Pentney, 2008). Knitting is no longer viewed as a necessary domestic pursuit, but a unique way to make thought provoking pieces. It also is a hobby practiced by celebrities who have been photographed knitting in a number of popular press publications which has helped to strengthen the revival (Parkins, 2004).

Another societal force impacting the resurgence of knitting is change in leisure. Increasing demands from the workplace have led a number of Americans to enjoy less leisure time than ever before (Arai \& Pedlar, 2003). Due to the limited time available for recreation, many Americans have redefined effective use of leisure time. Formerly, leisure was designated as a predominantly individual experience, but now Americans are reaching out to each other to create both physical and virtual communities centered around shared interests such as knitting (Minahan \& Wolfram Cox, 2007).

One of the most impactful activities knitting communities and knitters participate in are various forms of activism (Pace, 2007). Knitters use their crafts to convey messages about the social and political world. They may also sell the fruits of their labors to benefits various causes (Pentney, 2008). Environmental causes have similarly benefited from knitters who have donated their time and creations to support the "green" movement (Pace, 2007). The popularity of knitting has caused many yarn retailers and knitting shops to cater to the environmental awareness of their patrons by offering selections of yarns made from sustainable fibers (Zawilinski, 2010). 
The past ten years have brought about significant changes in knitting and how society views the process and products created by knitters. There is noteworthy growth in the number of participants using the technique (Minahan \& Wolfram Cox, 2007) and yet there has been little scholarly attention to understand the motivations employed by knitters and products they create. Similarly, there has been a lack of research on young, female knitters who are some of the most avid participants in the craft.

\section{Purpose}

The purpose of this research was to examine in depth young (ages 18-30), female participants of knitting in terms of motivations for participation and material culture. The researcher sought to categorize these women in terms of motivations for participation in knitting. Uses and Gratifications Theory was employed to better understand their motives. The researcher attempted to classify these knitters into one of five categories of motivations for participation as suggested by Katz, Blumler, \& Gurevitch (1974), which were: cognitive, affective, personal integrative, social integrative, and tension release. Artifacts made by the participants were also examined using material culture methods defined by Smith (1985) to determine if there was a correlation between motivations for knitting and fabrication of the artifacts produced.

\section{Research Questions}

The following research questions were identified for this study:

1. What are the motivations, benefits, and meanings of knitting for young women? How do these relate to Uses and Gratifications Theory?

2. What types of products are young, female knitters constructing?

3. How do the physical artifacts that are produced by young, female knitters relate to their motivations for engaging in knitting? 
4. How do the physical artifacts that are produced by young, female knitters relate to their specific social and political culture?

\section{Objectives}

To investigate the previous research questions the subsequent objectives were defined:

1. Test the framework as proposed by Katz et al (1974) and determine if the five categories of Uses and Gratifications: cognitive, affective, personal integrative, social integrative, and tension release are appropriate for describing the motivations of young, female knitters.

2. Use a combination of exploratory methods (interviews, surveys, and material culture analysis) to define the motivations and benefits for young women to participate in the knitting process.

3. Determine how the knitted physical artifacts that are produced by young, female knitters relate to their motivations for engaging in the knitting process.

4. Discover how the physical knitted artifacts that are produced by young, female knitters relate to their specific social and political culture.

5. Understand the meaning(s) of the knitting process and final products created for young, female knitters.

6. Define how the knitting process and final products created meet the motivational and beneficial needs of young, female knitters.

7. Create a basis for future research on knitting and other textile handcrafts.

8. Contribute to the scholarly knowledge base by describing the complex relationship between young, female knitters, their motivations for participation in knitting, and the physical artifacts they create.

\section{Assumptions}

The following assumptions underlie the study and were made during the data collection stage of research: 
1. Participants were female knitters who are between the ages of 18 and 30 years old.

2. All artifacts were produced by hand knitting methods as opposed to machine knitting.

3. All artifacts were produced by the interviewee and can be physically examined and photographed during the interview process.

4. All artifacts were produced within the time period between the years 2008 and 2011.

\section{Scope and Limitations}

The subsequent scope and limitations were identified for this research project:

1. As a knitter and a feminist, the researcher acknowledges that she will bring past experiences, potential biases, and previous knowledge of the topic examined into the research process.

2. The collection of qualitative data is based upon the individual participants within the study; therefore the data that will result from this inquiry cannot be applied to all knitters meeting the specified participant criteria.

\section{Definitions}

The following definitions have been included to clarify the technical terms utilized in this research. Some of these definitions are based on terminology specific to knitting:

\section{Artifact}

includes all human-made or human-modified things,

which makes "art” a sub-category of "artifacts”

rather than a separate and distinct category (Prown, 2000, p. $x$ ). 
Bulky Weight Yarn

Cable

Color Work

Craft

DK Weight Yarn

Feminists a heavy weight yarn, usually 5-ply that is about twice as thick as worsted weight (Stoller, 2003, p. 18).

a raised group of stitches which are created by passing stitches from the left needle to a holder and altering the order of stitches (Colton, 1979, p. 310).

knitting with two or more colors in which designs are made on the face of the fabric and floats are on the back (Colton, 1979, p. 318).

a skilled manipulation of physical materials into new forms (Rosner \& Ryokai, 2009, p. 195).

a medium weight yarn, usually 4-ply, it is thinner than worsted weight yarn (Stoller, 2003, p.17).

hold the view that women are less valued than men in societies that categorize men and women into differing cultural or economic spheres. They also insist that these inequalities are not fixed or determined, and that women can change the social, 
political, and economic order through collective action (Gamble, 1999, p. 230).

Fingering Weight Yarn

Flat Knit

Garter

Knit in the Round

Knitivism 
Knitting

Lace Stitches

Lace Weight Yarn

Motivation

Rib Stitch

Sock Weight Yarn

process by which a single yarn is lopped through itself utilizing two needles to make a chain of stitches that intermeshes horizontally referred to as courses and vertically called wales (DonofrioFerrezza \& Hefferen, 2008,p. 295).

any fabric construction using openwork stitches (Colton, 1979, p. 304).

one of the lightest weight yarns available, usually 2ply.

inner or social stimulus for an action (Edwards, 1999, p. 19).

is produced by altering knit and purl stitches on one row and then purling the knit stitches and knitting the purl stitches on the new row. The result is a pattern of vertical ridges on both sides (Colton, 1979, p. 280).

a light weight yarn, usually 3-ply or 2-ply that is thicker than fingering weight. 
Sport Weight Yarn

Stockinette

Worsted Weight Yarn a medium weight yarn, usually 4-ply that is thinner than DK weight yarn and thicker than sock yarn (Colton, 1979, p. 270).

a fabric construction using knit stitches on one side and purls on the other, it creates a smooth surface on the knit side and a pebbly surface on the purl side (Colton, 1979, p. 280).

a medium to heavy weight yarn with 4-ply (Colton, 1979, p. 270). 


\section{CHAPTER TWO}

\section{REVIEW OF LITERATURE}

\section{Introduction}

Reasons for the rapid increase in participation in knitting have only begun to be identified. Knitting in the new millennium has morphed from the old "make do and mend” ideology into one that sanctions consumerism, activism, and feminism (Turney, 2009). Researchers have stated that some women in contemporary society have a desire to reconnect with their feminine heritage and traditional textile handcrafts, like knitting, are often a good vehicle for this process (Prigoda \& McKenzie, 2007; Turney, 2004). Knitting also allows enthusiasts to forge new identities, retreat from their busy lives to indulge in an artistic pursuit, and to develop interpersonal relationships (e.g., SchofieldTomschin \& Littrell, 2001; Strawn, 2007; Turney, 2009). The friendships that are built among knitters and the value placed on the unique items they make help to build self worth (Campbell, 2005; Schofield-Tomschin \& Littrell, 2001).

A number of issues surrounding the revival of knitting will be discussed in the literature review. First, the history of knitting will be explained followed by the personal benefits of knitting, the meaning of knitted artifacts, the social benefits of knitting, the visibility of knitting, and the craft marketplace including a materials section. The 
theoretical framework Uses and Gratifications Theory and material culture studies will also be highlighted in the literature review.

\section{History of Knitting}

\section{Early Knitting}

Knitting is a very old technique which utilizes two needles. The first extant example of true hand knitting originated in Islamic ruled Egypt and has been dated to 600 A.D. (Rutt, 1987). The next major archeological find was a pillow with a complex repeating pattern knitted into the fabric discovered in a Spanish burial tomb from the late thirteenth century (Harris, 2004).

Even more extant textiles and garments were discovered throughout the early medieval ages. Many of these textiles were complex in pattern and construction, demonstrating that even during the medieval ages knitting was quite advanced. Various depictions of Madonna knitting appeared in medieval paintings, such as those by the Lorenzetti brothers, starting in the fourteenth century (Rutt, 1987).

Knitting was introduced as part of the educational curriculum for children starting in the sixteenth century; it was seen as a positive activity as it prevented idleness (Macdonald, 1988). The technique was taught to both boys and girls at this time. Children would knit while walking to and from school as well as in the evenings when there was little to do (Strawn, 2007). The items produced by the children could then be utilized in the home or sold to other families for a small price (Macdonald, 1988).

\section{Framework Knitting}

Although hand knitting was a popular mode of garment production throughout the medieval ages it did have the inevitable drawback of being time consuming and not 
particularly easy to standardize and industrialize. Demand for silk and woolen stockings increased during the sixteenth and seventeenth centuries (Donofrio-Ferrezza \& Hefferen, 2008). This led to the invention of the frame work knitting machine in the seventeenth century (Harris, 2004). Framework knitting machines made flat fabrics which were then cut and sewn into stockings (Rutt, 1987). Framework knitting solved the previous problems with hand knitting as it allowed for numerous stockings to be made faster.

The original framework knitting machine was capable of producing a plain knitted fabric that was very serviceable (Rutt, 1987). It was up-graded over the eighteenth and nineteenth centuries allowing producers to create more fabric faster, more complex patterns, and fabrics that were knit on the warp as opposed to the traditional weft knitting method (Donofrio-Ferrezza \& Hefferen, 2008). Circular knitting machines, which could create stockings without having to use cut and sew methods, began to replace the older version of framework knitting in the late nineteenth century (DonofrioFerrezza \& Hefferen, 2008).

In 1995, the Whole Garment knitting machine was unveiled (Donofrio-Ferrezza \& Hefferen, 2008). This machine knitted nearly complete garments that required only a few seams to be sewn (Donofrio-Ferrezza \& Hefferen, 2008). The WholeGarment technologies allowed for greater consumer wearing comfort and better fit than traditional cut and sew methods (Choi \& Powell, 2005).

\section{Knitting in America}

When people begin to colonize North America they brought their knitting with them. Knitting continued to be part of a proper education for both boys and girls. Children were even encouraged to knit during lectures, though this practice was later 
abandoned as teachers grew tired of having to stop lectures to aid the children when they dropped a stitch or needed help untangling mistakes (Strawn, 2007).

As the colonies began to work toward independence and boycotted English textiles, colonists took up the slack, carding, spinning, weaving, and knitting for independence (Macdonald, 1988). When the Revolutionary War broke out in 1775, the women of the colonies further increased their textile production, including knitting, to provide adequate clothing for the soldiers (Macdonald, 1988).

After gaining independence the United States sustained its fascination with knitting, which continued to be a key part of production and education for citizens. Knitting frames were smuggled into America in the mid eighteenth century and the knitting industry began to grow around the increased capacity to turn out knitted objects (Macdonald, 1988). The knitting industry developed and eventually grew large enough in the mid 1800s to provide many of the simple knitted items such as socks that were required on a day-to-day basis (Rutt, 1987).

\section{Victorian Fancy Knits}

The knitting industry freed women from having to use their knitting needles to make simple clothing staples like socks. This gave them more time to create extravagant items with complex patterns and stitches (Macdonald, 1988; Rutt, 1987). Although, it was not yet considered a strictly feminine pastime, knitting was emphasized as an important domestic art for women during the Victorian era (Turney, 2009). Patterns, needles, yarn, and other supplies began to be more readily available at stores and through catalogs, so women could plan and execute complex designs and showcase their skills (Strawn, 2007). A myriad of knitting magazines and manuals featuring patterns for 
garments such as bicycling sweaters and corset covers were published between the 1860s and 1900 (Macdonald, 1988). At this time knitting started to be identified as one of the key domestic skills for women to possess (Turney, 2009).

However, not all women at this time were interested in working on complex knitting patterns. During the nineteenth century the first wave of feminism began. These first feminists argued that many of the "apparent" differences between men and women were actually socially constructed (Arneil, 1999). First wave feminists were primarily concerned with issues of education, employment, and marriage laws (Gamble, 1999). In addition, the women in the movement were very concerned with suffrage for women (Gamble, 1999).

\section{Knitting for the Troops}

Knitting continued into the twentieth century and when the United States became involved in the first World War, women were asked to pick up their needles and knit for the troops (Strawn, 2007). Groups such as the Red Cross and Daughters of the American Revolution set up knitting contests, knitting circles, and even offered free supplies and lessons to knitters to make garments for the soldiers (Macdonald, 1988; Strawn, 2007). Knitting was shown as crucial and one of the best ways for women to aid in the war effort. Women latched on to this idea and knit constantly; they knit at the movies, during lunch breaks, and even while waiting for the bus (Strawn, 2007).

This is not to say that men were not knitting for the troops as well. In fact, there are records of some exceptional male knitters who created numerous garments to be donated to the war effort (Macdonald, 1988). It was also common for injured soldiers 
and even some on duty to knit while waiting for the next round of fighting (Macdonald, 1988).

\section{The Roaring Twenties and Knitting}

With the end of World War One, there was less need to knit at the same furious pace as during wartime. Major social changes began to occur within American society during the 1920s, women had gained the vote and began to move out of the home and enter the workforce. The new sense of freedom women felt may have led them away from knitting and other homemaking chores (Macdonald, 1988).

Knitting suppliers had to get creative to re-interest American women in knitting. They started offering patterns for fun fashion-forward garments, patterns with more complex and challenging stitches, and stocked an array of new yarn colors (Strawn, 2007). The yarn companies offered various contests for knitting such as Star Needlework Journal’s \$2,000 prize for the best original knitted design (Macdonald, 1988).

\section{Knitting Through the Forties and Fifties}

The outbreak of World War Two produced another call for knitted garments for the troops (Macdonald, 1988; Rutt, 1987; Strawn, 2007). Women picked up their needles and knitted with the same fervent nature as they did for the First World War (Macdonald, 1988). Women also took over much of the production of goods and other jobs while the men were away fighting. Icons such as Rosie the Riveter were used to encourage women to leave the home and do their part to help the war effort (Strawn, 2007).

A second propaganda campaign was launched after the war to persuade women back into the home because the jobs that they had taken during the war were needed for the troops returning from overseas (Rutt, 1987). The concept of being a good 
homemaker and mother was extolled and women were encouraged to find satisfaction in being able to provide meals, a clean home, and proper clothing for their families (Arneil, 1999). Knitting played into this ideal housewife image as it was aligned with productivity and thrifty housekeeping (Macdonald, 1988).

\section{Feminism Calls for the End of Knitting}

Social and political unrest during the 1950s led to a number of social changes in American society. One of the most powerful movements at this time was the second wave feminist movement. Second wave feminists pointed out flaws in the social structure. They believed being content to be both a housewife and mother was at odds with women's ability to become truly independent (Arneil, 1999). Betty Friedan's book, The Feminine Mystique (1953) was one of the first publications to point out that trying to be a perfect wife and mother was in conflict with being personally content. She suggested that the solution was for women to expand their lives beyond the private/domestic sphere and begin seek education and enter the workforce (Arneil, 1999). This wave gained movement and grew in power through the 1960s. During this time women fought for and won numerous battles over fair wages, equal opportunities in education and more (Gamble, 1999).

After the feminist revolution in the 1960s, knitting was largely discouraged because of its fundamental ties with housewives (Stoller, 2003). Women were expected to cut ties with anything that connected them to the image of the homemaker. Feminists viewed knitting and other typically female chores as thankless tasks that kept women tied to the low status role of a housewife (Gamble, 1999). They felt domestic tasks were also standing in the way of work and educational opportunities for women (Arneil, 1999). As 
knitting was so tied to the idea of the 1950s housewife, it was largely discouraged and suffered a huge decline between the 1960s and 2000 (Strawn, 2007).

\section{September, 112001 and the Resurgence of Craft}

Knitting reemerged in the twenty first century as a trendy occupation (Strawn, 2007; Turney, 2009). The reasons for this come back are varied and complex. Many researchers have pinpointed the terrorist attacks on the World Trade Center in New York City on September 11, 2001 (9-11) as one of the causes for the reemergence of knitting.

\section{9-11 created a social backlash in America which left Americans feeling}

vulnerable and in need of new methods of escaping from stress (Wayment, 2006). Many Americans reported feeling psychiatric symptoms from distress to more severe symptoms that can be connected to post-traumatic distress disorder (Wayment, 2006). Researchers who have studied the social changes following 9-11 have found some interesting trends. For example, Stein, et.all (2004) discovered that depending on how deeply affected people were by the attacks they engaged in behaviors such as turning to religion, increased volunteerism, seeking meditative opportunities, and spending more time at home. Americans turned to leisure pursuits that provided occasions to do any of the above activities.

Traditional textile handcrafts were one of the leisure pursuits adopted by American citizens in response to 9-11. These traditional textile handcrafts include: knitting, quilting, crochet, bead work, and embroidery. It has been suggested that crafting is appropriate for leisure time because it allows participants to create unique items, to multi-task on other projects, and to engage in community building activities around the shared interest in crafting (Arai \& Pedlar, 2003; Campbell, 2005). 
The change in knitting has also been linked by a number of researchers to feminist ideology (i.e., Parkins, 2004; Pentney, 2008; Turney, 2009). However, there does not appear to be a clear choice of what type of feminism the new knitting relates to. The rejection of the dualistic system (men verses women) and other ideas about the perceived need for the feminist movement led to a number of new philosophical perspectives within feminism. Anti-rational and third-wave feminism are two of these emergent feminist perspectives, which seek to revalue and celebrate traditional women's practices such as knitting (Arneid, 1999).

\section{Personal Benefits of Knitting}

\section{Psychological}

Knitting has a number of advantages to offer participants. The soft clicking of needles and flow of the yarn into patterns has been described as an alternative meditative process (MacDonald, 1988). Knitting has been endowed an almost Zen-like quality that allows for the individual to retreat from a hectic lifestyle to focus on a simple, creative process (Parkins, 2004). Indeed, this quality has sparked the imagination of authors to create books such as, Zen and the Art of Knitting (2002), The Knitting Sutra (2004), and Mindful Knitting (2004).

Another major psychological advantage of knitting is the creation of self. Knitting and other traditional textile handcrafts have been found to help create a sense of identity for the crafter for example, “I am a quilter” (Hawley, 2005; Schofield-Tomschin \& Littrell, 2001). Identities such as being a quilter are particularly valued in contemporary society as so few individuals have the ability to create homemade objects (Hawley, 2005). Similarly, knitting allows women to connect with their feminine 
heritage and practice the traditions that have been passed down from mother to daughter for generations (Myzelev, 2009).

\section{Physiological}

In addition to the meditative benefits, there are physiological changes that occur while one knits; a decrease in heart rate, slower breathing, and increased manual dexterity (Prigoda \& McKenzie, 2007). Due to these positive effects knitting has been explored as a therapy medium for individuals suffering from a wide array of conditions ranging from depression to chronic illness, and aging (Reynolds, 1997; Reynolds, 2009; SchofieldTomschin \& Littrell, 2001).

A link has been made between caring for the self and increased physical wellbeing (Reynold, 2009). Today knitting is done not because it must for the family to have socks, but because the knitter wants to practice it as a hobby. This can be seen as caring for the self by focusing on something that brings simple pleasure and perhaps does not have certain outcomes that help the family unit (Hollows, 2003). Knitters claim their hobby is also an excellent stress reliever (e.g. Parkins, 2004; Stoller, 2003).

Knitting has become a preferred leisure time activity because it allows for multitasking as well (Myzelev, 2009). Knitters can work on a project while listening to music, carrying on a conversation, or watching television. The amount of leisure time available has become significantly less than in previous generations so activities that allow for simultaneous leisure activities to occur are particularly appealing (Arai \& Pedlar, 2003).

\section{Meaning of Knitted Artifacts}

\section{Creativity through Knitting}

Knitting gives women an outlet to showcase their skills, personal aesthetics, and 
creativity (e.g., Hawley, 2005; Johnson \& Wilson, 2005; Schofield-Tomschin \& Littrell, 2001). Although patterns and kits are often utilized, many offer choice of yarn or color. The chance to choose colors and patterns gives a greater sense of participation in the design on the object, which, in turn, bolsters the makers' sense of pride upon completion (Hawley, 2005; Johnson \& Wilson, 2005; Schofield-Tomschin \& Littrell, 2001).

Even mistakes are embraced as they allow slight changes in the overall look of the product. Handmade products and processes are especially well regarded because they represent the unique, singular, and self-expressive (Campbell, 2005). Uniqueness is especially valued in postmodern society where due to globalization and mass production many products are homogenized (Morgado, 1996). Hand knit clothing can also overcome the limited sizing and fit possibilities that are a major concern with mass produced items (Chansky, 2010).

\section{Knitted Gifts}

The products created by knitters hold specific roles in the lives of their creators. Many women talk about strong emotional bonds with on-going projects (Hunt, 2005; Johnson \& Wilson, 2005). These projects upon completion can be presented as gifts to family and friends, donated to charity, or sold. According to Reynolds (1997), "many respondents had found that their needlework provided them with an expanded social role and a means of making a 'useful' contribution (in their own eyes) to their families or wider community"(p. 354). The ability to contribute to a good cause or the family coffers further strengthens the crafter's resolve to continue to produce home-made items.

Handmade items have become extremely popular in postmodern society and are also being sold by a number of websites such as etsy.com (Campbell, 2005). These 
websites allow non-knitters to obtain hand knit clothes and accessories to use in their everyday lives. One business that has been created to fill this niche is the GranniesInc Company. Their website invites customers to log in and create their own knitwear design and then choose a granny to knit the product (Skinitis, 2009).

\section{Social Benefits of Knitting Circles}

\section{Camaraderie}

When women and men participate in knitting outside their home environment they are often said to be members of Stitch ' $n$ Bitch. Practitioners who band together to "Stitch 'n Bitch" discover positive social benefits like the ability to spend time with other crafters, and learn new skills. Stitch ' $n$ Bitch (Stoller, 2003) is a book that encourages readers to get out of the house and knit.

The opportunity to exchange ideas and socialize with other crafters is particularly important to individuals who may have limited chances to interact with others (Reynolds, 1997; Reynolds, 2009). Knitting is seen as being nostalgic, perhaps yearning for a time when communities were more inter-connected and spent time socializing together (Myzelev, 2009). The desire for a more interconnected community has been discussed as one of the main forces behind participation in leisure activities (Arai \& Pedlar, 2003).

\section{Social Support}

Passing on techniques, which can be seen as generativity is extremely important to members of crafting groups (Hawley, 2005; Schofield-Tomschin \& Littrell, 2001). Many women are compelled to teach other members of a guild or knitting circle new stitches and methods of construction to help keep the tradition alive. This is crucial today because so many new knitting enthusiasts have learned from the Internet and books as 
opposed to their mothers or grandmothers, which can make it difficult for them to get help on their projects (Parkins, 2004). By engaging with newcomers and practicing generativity, members can reinforce their identity as not only a knitter, but also as a teacher.

Participants in Prigoda and McKenzie’s (2007) study of a library knitting group described how the circle had become a social support group for many of the knitters. A number of topics arose in the knitting group such as funeral arrangements, illness, religion, and more that participants may not have been able to discuss with other firends. Being able to unburden themselves to other members of the knitting circle allowed the knitters to feel even more connected and committed to keeping the group going.

\section{Social networking}

A number of social networking websites dedicated to knitting have cropped up since the year 2000 (Minahan \& Wolfram Cox, 2007). These sites are committed to celebrating knitting by showcasing finished designs, offering advice, and connecting knitters (Stoller, 2003). Websites such as www.ravelry.com list knitting groups by geographic location so knitters can log on and find a group to join in their hometown (Minahan \& Wolfram Cox, 2007). Online groups allow knitters who may be geographically isolated or physically incapable of attending physical meetings to enjoy the positive outcomes from participation (Reynolds, 2009; Rosner \& Ryokai, 2009). Knitters can also choose to send messages through these sites to other crafters creating extended communities. Many knitting websites allow enthusiasts to sign up for newsletters so they can receive e-mails about new patterns, troubleshooting, and designs ideas (Stoller, 2003) 
The trend of on-line knitting knowledge models how technologically savvy today's crafters are. Some researchers have pointed out that handheld devices such as Ipods ${ }^{\circledR}$ and Blackberries ${ }^{\circledR}$ require manual dexterity similar to that which is required to knit; therefore knitting can be seen as an extension of these same skills which are already used in modern society (Minahan \& Wolfram Cox, 2007). Additionally, many knitters have opened Internet-based businesses utilizing websites like www.etsy.com to sell finished products, home spun yarns, and patterns. These cyber businesses further showcase a comfort with technology among knitters (Chansky, 2010).

\section{Visibility of Knitters}

\section{The Celebrity of Knitting}

Recent articles in the popular press have shown celebrities, such as Julia Roberts knitting (Eig, 2002; Parkins, 2004). Other stars have began to frequent various high-end yarn shops such as La Knitterie Parisienne where they have been photographed indulging in their new hobby (Eig, 2002). There are even celebrity lines of knitting supplies such as Vanna White’s yarn line and the book Hollywood Knits (2007).

The interest of celebrities takes knitting to a new level of social acceptance (Minahan \& Wolfram Cox, 2007). Parkins (2004) added that the sight of celebrities, who are seen as extraordinary people, knitting elevates the process from a typical and possibly even antiquated craft to an extraordinary hobby. It has been suggested that since celebrities have been sighted on-set, in cafes, and outside the home, it may have led regular knitters to experiment with publically performed knitting (Parkins, 2004). 
By taking their crafting into the public arena, knitters are forging a new identity not only for themselves, but for knitting in general. When looking at public knitting Parkins (2004) stated,

the urban café, as a space of vibrant and visible sociality in modernity where the exchange of (the latest) ideas can take place, is represented as a site for (new) knitting which takes on a public dimension not traditionally associated with the craft (p. 430).

These public spaces are known as third place, which is essentially anywhere outside the home or work environments (Minahan \& Wolfram Cox, 2007). By claiming these public spaces for a craft that is associated with the private sector, knitters are demonstrating knowledge of the societal rules that govern public spaces and a desire to change the rules about the space and the rules that place knitting outside of the public eye (Medford, 2006).

The use of third place as a location for knitting coincides with the concept that knitting is now a hobby of consumption. When knitters take their places in a café or bar they will need to purchase a beverage to justify their occupation of the space (Turney, 2009). This is evidence toward the idea that knitters are no longer housewives trying to stretch a dollar, but career women happy to purchase fancy yarns and five dollar lattes to support their hobby (Parkins, 2004).

\section{Knitivism}

Knitting has been adopted for social, political, and environmental activeness. Activists seek to bring about change for various causes such as: social, political, and environmental (Gamble, p. 186, 1999). When knitters use their work for a specific social cause, it is called knitivism (Pace, 2007; Springgay, 2010). Sometimes knitivism is 
organized by large national groups such as the Red Cross, but it is also done on a small group or individual scale (Minahan \& Wolfram Cox, 2007).

One example of knitivism for social change was the “Afghans for Afghan” project (See Appendix A) undertaken by a small university based group (Minahan \& Wolfram Cox, 2007). They created large knitted blankets called Afghans (Pace, p. 17, 2007) and sent them to Afghanistan staring in 1999 (Pace, 2007). Other groups have organized "knit ins" where participants meet at specific locations and spend time knitting for causes such as knitting for the troops (Pentney, 2008). Participants of a "knit in” which was used to protest violence in Darfur were particularly pleased with the peaceful nature of knitivism. It is in stark contrast to the raised, angry voices of marches and rallies often associated with social protest (Springgay, 2010). Other examples of knitivism include: knitting caps for premature babies (See Appendix A) and various groups focused on knitting warm clothing for children in third world countries (Pace, 2007).

Clearly, knitting has been used as a vehicle for enacting social change. In addition to these causes, knitting has been used to protest and comment on politics. Some knitters have used their skills to create large scale art objects for this purpose. One example is the knitted tank cover by Marianne Jorgensen (See Appendix A) used to protest Denmark's involvement in the Iraq war. The artist created thousands of pink knitted squares which she sewed together and then covered a World War II military tank with (Pace, 2007; Pentney, 2008).

According to Pentney (2008) the Wombs on Washington project (See Appendix A) was another example of political knitivism. The participants of this project made knitted wombs using a pattern that could be obtained on the project's website. The 
project organizers planed for the wombs to be taken to Washington D.C. and thrown on the capitol steps to protest a change in abortion law. While the wombs never made it to Washington, it is certain that they are an example of knitivism.

Finally, knitting has been adopted for environmental activism. Knitters have used their projects to generate funds for various environmental causes. One example was the Knit for Water project which used blue knitted squares to create a knitted river in downtown London (Pace, 2007). The river visually represented the lack of clean drinking water around the world and onlookers were asked to donate to the cause. Another group in 2004 used their knitting prowess to create miniature knitted sweaters for Australian fairy penguins (See Appendix A). These sweaters kept the penguins from preening their feathers to a point of destruction after they had been coated in oil from a large oil spill (Pace, 2007).

Using knitting to enact change in the world has become an extremely important part of many knitters' experience of the craft. Participants in these events are steadily growing in numbers perhaps because of the increasing availability of connections through knitting groups and on-line knitting forums (Pentney, 2008). By using their skills to help the world knitters are showing what social, environmental, and political causes are most important in their lives.

\section{The Craft Marketplace}

\section{Knitting Supply Retailers}

A number of businesses have evolved to uphold and further the knitting craze. These retailers do offer traditional supplies like yarn and needles, but they provide much more for the knitter. Some of the services may extend to free knitting lessons or 
established knitting groups patrons can join (Parkins, 2004; Strawn, 2007). Yarn shops are even including espresso stands and cozy "knitting nooks” so knitters can be comfortable while shopping or participating in in-store knitting groups (Strawn, 2007).

Stoller (2003) has created the acronym of "SEX" to stand for what she terms as a "stash expanding expedition." These trips are not for gathering materials for specific projects, but for simply going and purchasing whatever materials look appealing. This implies that lingering in the store and shopping for nothing in particular are a regular part of the new knitting culture (Strawn, 2007).

\section{Materials}

The reemergence of knitting over the past decade has brought about not only surprising changes in participants, but also a revolution in the materials employed to create projects. According to Karen Searle (2008) in her book Knitting Art, there has been a surge in the number of artists who are interested in traditional textile techniques to create art objects. These artists are pushing the boundaries of shape, stitches, and also materials for knitting. Some of the alternative materials that have been used by artists to knit include: metal, plastic, paper pulp, bark, and many other "found” objects.

The shift in materials has not been exclusive to the artist community; everyday knitters are also seeing an expansion in available yarn options. According to yarn.com (2010), yarns made from exotic fibers such as: vicuna, angora, camel, and bison are now commonplace and ready for purchase at some of the county's premier knitting retailers. Similarly, there are also a number of books to teach knitters how to use alternative materials in their knits such as Knitting with Wire (2003), Knitting with Dog Hair (1995), and Knitting with Novelty Yarns (2001). 
The expansion of available yarns includes an effort to market sustainable yarns for knitters. These yarns are made from various fibers such as: bamboo, corn, soy silk, hemp, recycled cotton, organic cotton, and even seaweed (Zawilinski, 2010). Many of these yarns not only offer a sustainable product, but also include information as to charitable causes that knitters are aiding by purchasing their yarn (Strawn, 2007). Some companies offer yarns made from recycled clothing such as saris and sarongs (Zawilinski, 2010). Yarns being made from recycled garments have been popular among knitters because of their sustainability and contributions to the countries in which they are produced (Strawn, 2007).

There are a number of reasons why young people are inclined towards purchasing sustainable goods. Consumers of sustainable or "green" products are described as well educated with an income that allows for the purchase of slightly more costly green products (Ogle, Hyllegard, \& Dunbar, 2004). In their study of green consumer behavior, Autio, Heiskanen, and Heinonen (2009) found many young people today have grown up in a social climate that promotes green consumption which makes purchasing along a sustainable path normative. While there are still large parts of the population not practicing green consumer behavior it is no longer difficult to find green goods at even the most mundane retailers.

Today there are boundless choices when it comes to buying yarn for knitting. The sheer number of types of yarn showcases just how versatile knitters are in their uses of materials. It also showcases that many knitters are aware of the impact their consumer decisions have on the environmental and social world. The availability of sustainable 
yarn confirms the growing trend of green products and the comfort young people have with these items in their daily lives.

\section{Knitting Publications}

Knitting publications known as "knit-lit" have undergone a significant expansion in the past ten years (Myzelev, 2009). These books range from novels to quasi-religious stories, and even directions on how to create knitted objects for erotic purposes. These publications glorify knitting, knitters, and enforce the new relationship that exists between the craft and its participants. With so many types of "knit-lit" available it is a logical to believe that there are many types of knitters who are buying them.

While traditional instructional manuals and pattern books are still popular, the appearance of other knit-lit is surprising. Certainly knitted items for "the bedroom" would not have appeared before the revival of knitting. This phenomenon has been suggested as proof that feminists are seeking to reclaim their domestic heritage and reevaluate crafts while embracing a level of comfort with sexuality previously associated with men (Turney, 2009).

\section{Theoretical Frameworks and Data Collection Process}

This study is exploratory in nature, there has been very little research in this area and no research has provided a framework that can be easily used to describe the motivations of young, female participants of knitting. In order to understand these women the framework, Uses and Gratifications Theory and material culture process was used. 


\section{Uses and Gratifications Theory}

Uses and Gratifications Theory (UG) is grounded in the field of mass media studies. UG was originally developed in an attempt to describe the various reasons people sought out and used mass media including radio, newspapers, books, and eventually television and the Internet (Severin \& Tankard, 1997). One of the primary assumptions of UG states that the audience must be viewed as active participants who seek out various media sources in order to satisfy needs (Eighmey \& McCord, 1998; Katz, Blumler, \& Gurevitch, 1974). UG has been utilized by researchers to describe interactions between people and mass media for example: online shopping preferences (Cho, 2007), e-learning (Mondi, Woods, \& Rafi, 2008; Severin \& Tankard, 1997), and advertising effectiveness (Ko, Cho, \& Roberts, 2005).

Researchers have argued mass media can be used as a leisure activity to meet a number of human needs. However, the categories of needs identified by researchers do not necessarily coincide. For example McQuail, Blumler, and Brown (1972) advocated four categories of needs that could be fulfilled using mass media; diversion, personal relationships, personal identity, and surveillance. Those seeking to fulfill diversion needs used mass media to escape from reality and provide entertainment. Personal relationship needs referred to the desire for strengthening bonds between family and friends. Personal identity needs centered on the need for value reinforcement and self exploration. Finally, surveillance needs referred to the desire to be informed about current situations which may affect an individual.

The work of Katz et al (1974) identified five types of needs which could be met using mass media. These categories were: cognitive, affective, personal integrative, 
social integrative, and tension release. Cognitive needs referred to the desire to seek out information, knowledge, and understanding. Affective needs discussed the need for emotional fulfillment, pleasure, and aesthetic experience. Personal integrative needs focused on credibility, confidence, and status. Social integrative needs referred to the desire to seek out stronger bonds with family and friends. Tension release or entertainment needs referred to the need for soothing, diversion, and calming.

These are the two most frequently used sets of categories within the UG (Severin \& Tankard, 1997). Clearly there is significant overlap between some categories within the two traditions. Consider the categories of diversion and tension release, both are used to describe how media may be used to provide escape and entertainment. Similarly, the categories of personal relationships and social integrative describe how mass media can be used to strengthen intrapersonal bonds.

Differences between the two sets of categories are significant. For example surveillance describes a desire to be informed on current issues and personal integrative needs also use mass media to be up-to-date on issues. However, the personal integrative needs call for credibility and status taking the surveillance needs further by describing outcomes a person may gain with their knowledge of current issues.

McQuail et al‘s (1972) category of personal identity, which reinforces personal values and provide opportunity for self-exploration also differs. Katz et al (1974) split this category into both cognitive and affective needs allowing for the identification of those using mass media for more practical knowledge and understanding as opposed to those seeking a more emotional and aesthetic experience. 


\section{Application to present study.}

UG came from the mass communications field and had not been applied to studies about participation in crafting. However, it had been used to describe interactions between people and the Internet (Ko et al., 2005) as well as how mass media may be used recreationally (Severin \& Tankard, 1997). Knitting is very connected to media; there are numerous knitting publications as well as the countless number of online knitting resources (Turney, 2009). Knitting is no longer a required household chore; therefore participants must have had motivation(s) for deciding to participate in it. The UG framework could be used to understand what motivated young women to participate in knitting.

Thus, the researcher believed each UG category could be used to describe different types of motivation for participation in knitting. The five categories as defined by Katz et al. (1973) were chosen over the work of McQuail et al. (1972) for use in this study primarily because the categories described by Katz et al. were more inclusive and closely aligned with the potential uses of knitting. In addition, other researchers have had trouble duplicating the categories as describe by McQuail et al. (Severin \& Tankard, 1997).

Those seeking to fulfill cognitive needs may have participated in knitting because they were trying to understand how the process of knitting works. Knitting is math and counting based, one must consider the number of stitches per inch and sometimes make mathematical adjustments to commercial patterns (Parkins, 2004). Further, knitting and other traditional textile handcrafts have been linked to mathematics and are suggested as 
visual aids in teaching by a number of researchers (Belcastro \& Yackel, 2008; Harris, 1988).

Participants who were knitting in order to fulfill affective needs may have done so out of a need to be creative. Individuals who become engrossed in their creative work can enter into a flow state (Csikszentmihalyi, 1990). While in a flow state individuals may experience intense feelings of pleasure and motivation to continue with the activity at hand. However, it is easy to become bored with leisure activities that had once induced flow so participants often seek out new skills and challenges within their chosen activity in order to prolong flow and enjoyment of their work (Csikszentmihalyi, 1990). The feelings of pleasure that can result from creative engagement in knitting may have motivated knitters to seek out new creative challenges within the activity. Flow has been used to explain the motivations and outcomes of those who create garments using their own design and sewing skills (Blood, 2006; Engel-Enright, 2007). As knitting allows for the same exploration of design and materials, it may be possible that knitters were entering into a similar flow state.

Those who knitted in order to satisfy personal integrative needs may have used their skills to gain status within the knitting community. The Internet created the opportunity for validation of a participant's work and ideas by other knitters (Minahan \& Wolfram Cox, 2007). Blogging was one specific activity knitters could use in order to gain creditability as a knitter and status within the online knitting community. These participants may have used their online ventures as a way of gaining status by counting how many visitors they had to the website or perhaps how many messages were posted 
about their work. Another way knitters could gain status through their craft was by their use of expensive exotic materials such as vicuna, cashmere, and silk (Parkins, 2004). Social integrative needs may have motivated participants to knit for a number of reasons. As stated earlier there are many different social opportunities for knitters. They can join physical knitting groups, seek out knitting causes to participate in, and there are numerous of online knitting forums, chat rooms, and cyber knitting groups to take part in. The ability for women to build communities around a shared hobby has also been cited as one of the goals of feminists who have adopted knitting and are attempting to have the activity revalued within society (Parkins, 2004; Stoller,2003).

Those who had tension release or entertainment needs may have sought to participate in knitting because of its stress-relieving and meditative qualities. Many participants have described how knitting allows them to calm down and enter into a meditative state (e.g. Medford, 2006; Minhan \& Wolfram Cox, 2007; Turney, 2009). The popular press has supported this trend and various camps and organizations are now offering retreats for enthusiast to enjoy meditation, knitting, and even yoga (Turney, 2009).

\section{Material Culture Studies}

Material culture as defined by Prown in his introductory article is "the study through artifacts of the beliefs-values, ideas, attitudes, and assumptions- of a particular community or society at a given time” (1982, p. 1). Essentially, material culture utilizes the artifacts of a culture as a way to point toward or imagine the mentifacts and sociofacts of that same society. Prown (1982) argued that the use of artifacts to interpret a culture is 
the most sincere method of interpretation and is applicable to a larger portion of the population because objects are part of the everyday lived experience.

Smith (1985) presented another method for the study of material culture. He used an ongoing dialog between himself and his students to create an appropriate method for approaching material culture. The five major categories developed by Smith for describing artifacts were: material, construction, provenance, function, and value.

The material category examined the physical materials such as metal, glass, fibers, employed in the creation of the artifact. The construction category looked at the ways the materials were joined together in the artifact, for example the joints in furniture or the seams in a dress. Provenance examined the ownership of the artifact as well as the original producer. The function category provided information on the possible uses of the artifact and the effectiveness of the artifact for specific functions. Value referred to the holistic value an artifact may have. For example an artifact may have emotional and societal worth in addition to monetary value.

Each of these categories was examined in three stages of analysis. The first stage recorded only observable data, the researcher would write what types of materials were used to create the artifact being studied, what sorts of construction techniques were employed, and so on. The second stage of analysis was titled comparable data. This stage had the researcher compare the artifact being studied with other similar artifacts. A supplementary data stage allowed the researcher to find additional sources such as books to better understand the artifact. This was followed by a general conclusions section where the researcher would sum up what the artifact was and what it meant to the specific culture that created it. 


\section{Application to present study.}

Research on knitting tends to focus on the physical process such as how knitting can be beneficial for social reasons. However, one cannot ignore the actual artifacts being produced by knitters. To better interpret and understand the artifacts this research used material culture methods. The categories outlined by Smith (1985) were filled with knitting terminology to create an instrument to study knitted artifacts. This was very useful as the physical artifacts created by knitters were infused with a myriad of cultural meanings that had yet to be explored by the academic community.

\section{Relationships of Theoretical Frameworks and Data Collection Process}

As there are no previous scholarly studies that have sought to examine both the motivations for participation in knitting and the physical objects produced by knitters it is difficult to say if there will be overlap between the two theoretical frameworks. For example it is possible that a knitter may use the hobby for social integrative reasons and because of involvement with a knitting circle they may create a project that reflects the tastes of the group. It is also feasible that there will be no overlap at all. One of the aims of this study will be to determine and describe the relationship between UG and material culture in the context of knitting.

\section{Critical Evaluation of Existing Work}

The subject of knitting as a feminine tradition and a trendy leisure activity has only begun to be examined by researchers. Many of the articles that inquired into this topic were purely descriptive. If data were gathered it was by qualitative means such as participant observations and interviews. Oral histories were also used to examine trends in knitting and craft. 
The participants were almost always crafters, only one article interviewed noncrafters to provide a foil to the crafter perspective (Turney, 2004). Nearly all the crafters who were studied were women and they were often found at guild or sewing circle meetings. There were some studies that included perspectives of younger participants, but as a whole middle aged and older crafters were focused on (i.e. Hunt, 2005; Medford, 2006).

Theory and models were infrequently used, two studies used a theory to shape their research, of these one article managed to develop a model after collecting data (Hunt, 2005; Schofield-Tomschin \& Littrell, 2001). Feminist theory was often mentioned within the research, but was rarely employed to shape the data collection or analysis.

To improve the body of knowledge on this particular topic, researchers should employ other types of data collection. There is a need for understanding younger knitters as they are some of the most enthusiastic participants in the craft (Schofield-Tomschin \& Littrell, 2001; Stoller, 2003). It is important to study how young knitters relate their knitting to larger societal movements as well as political, social, and environmental causes.

In order to understand the specific cultural values of knitters, research must be done on the physical artifacts being produced by these women. The influx of new materials available for knitters to utilize had changed what types of projects knitters are able to create and may have an impact on the goals and designs of the producer. Knitted objects stand as markers for the mental and social standpoints of their creators and should be treated as valuable data. 


\section{CHAPTER THREE METHODS AND PROCEDURES}

\section{Introduction}

The purpose of this research was to examine in depth young (ages 18-30), female participants of knitting in terms of motivations for participation and material culture. The study sought to categorize these women in terms of motivations for participation in knitting. Uses and Gratifications Theory was employed to better understand their motives. The researcher attempted to classify the knitters into one of five categories of motivations for participation as suggested by Katz, Blumler, \& Gurevitch (1974) which are: cognitive, affective, personal integrative, social integrative, and tension release. Artifacts made by the participants were examined using material culture methods as suggested by Smith (1985) to determine if there is a correlation between motivations for knitting and fabrication of the artifacts produced. This purpose was explored in depth using the following research questions:

1. What are the motivations, benefits, and meanings of knitting for young women? How do these relate to Uses and Gratifications Theory?

2. What types of products are young, female knitters constructing?

3. How do the physical artifacts that are produced by young, female knitters relate to their motivations for engaging in knitting? 
4. How do the physical artifacts that are produced by young, female knitters relate to their specific social and political culture?

\section{Pilot Testing}

A pilot study was conducted to ascertain if the data gathered using the instruments would meet all the research objectives. The pilot test consisted of a single interview utilizing an interview schedule (see Appendix B) with a knitter and an analysis of an artifact she had produced. The material culture instrument, (Knitted Artifact Instrument), was also employed in the pilot study (see Appendix C). After the pilot study was completed the interview transcript and Knitted Artifact Instrument were examined by the researcher and advisor. No changes were made as elements were effective and produced the desired types of data for analysis.

\section{Sample Selection}

There has been a call in the research for a study that addresses younger participants of crafting, specifically knitting (Schofield-Tomschin \& Littrell, 2001). In order to attend to this discrepancy in the knowledge base, the researcher located a sample of fifteen female participants between the ages of 18-30. These women identified themselves as knitters and were of all skill levels ranging from beginning knitters to advanced knitters.

A couple of recruitment techniques were employed in order to find potential participants from the Northern Colorado area. A flyer asking for participants was posted at local yarn and craft stores (see Appendix D). A similar message was posted on www.ravelry.com, an online knitting forum. In an effort to speed data collection further 
participants meeting the criteria were identified by means of a snowball sampling method using previously identified participants.

\section{Data Collection}

The data collection stage began upon approval by the Human Research Subjects Committee at Colorado State University on January 26, 2011. Once approval was granted the identified research participants were invited to schedule an individual interview with the researcher. Each participant was asked to bring at least one knitted artifact they have produced for analysis by the researcher. The artifacts could be something that could be worn on the body, such as scarves, sweaters, socks, hats, and mittens. It could also be an artifact that was not meant to be worn such as an afghan, toy, or other decorative items. Following the interview and material culture analysis the participants were invited to fill out a Post Interview Participant Survey which was delivered via e-mail.

\section{Interviews}

The study began with a semi-structured interview with the participant (see Appendix B). The consultation employed an interview schedule to guide the data collection process. There were allowances for additional questions and discussion as they pertained to specific content. The conversations were recorded with a digital recorder to facilitate recovery of data and the analysis process.

\section{Knitted Artifacts}

The Knitted Artifact Instrument (see Appendix C) was developed from the methods of studying material culture as proposed by Stuart Smith (1985). Smith identified five main categories of inquiry when examining artifacts, material, 
construction, provenance, function, and value. Each of these categories was examined in three stages of analysis, observable data, comparable data, and supplementary data.

The knitted artifacts were photographed so the researcher could refer back to them in the data analysis stage. The observable data section was filled out jointly by the researcher and participant following the interview. The only section that was filled out during the interview was the observable data section.

After all interviews were concluded the researcher completed the additional sections of the Knitted Artifact Instrument starting with the comparable data section. The comparable data section was used to contrast between the sample of artifacts brought for inclusion in the study and knitted items created by a larger sample. The larger sample was obtained from www.ravelry.com which is a global, social network commonly used by knitters. It was ultimately chosen as it is the premier knitting social network. The website features patterns, yarns, and finished projects, all of which can be easily searched. It was possible to compare across the sample as the artifacts from the participants and the artifacts on www.ravelry.com because one can search for items with similar functions, materials, and construction (Smith, 1985).

Searches for comparable data were accomplished with large descriptive terms for the artifact such as "lace, scarf." These searches were done through the patterns section in order to find only finished items and because of easy to access information which allows users to look at fiber used, yarn weight, and other important aspects of the patterns. To make comparisons across the two samples it was necessary to limit the size of the comparable data projects. Therefore, the first project to appear for each search was utilized. 
Upon completion of the comparable data section, the researcher then consulted external sources which are displayed in Table 3.1, to locate information to aid in explaining the trends from the previous sections.

Table 3.1

Supplementary Data Sources

\begin{tabular}{|lll|}
\hline Author & Date & Title \\
Colton (Ed) & 1979 & Reader's Digest Complete Guide to Needlework \\
Hiatt & 1988 & The Principles of Knitting \\
Hollingsworth & 1982 & The Complete Book of Traditional Aran Knitting \\
Stanfield & 2007 & 150 Knitted Trims \\
Walker & 1971 & The Craft of Lace Knitting \\
\hline
\end{tabular}

This information was recorded in the supplementary data section. The final step in filling out the Knitted Artifact Instrument was to draw conclusions about the artifacts.

\section{Post Interview Survey}

After the initial stages of data collection it became clear that to best describe the participants, demographic data and additional information was needed. To collect this information a short survey was generated (see Appendix E). The survey asked for general descriptive data such as age, income, and education. A secondary portion of the survey asked questions relating to knitting such as how frequently knitting occurred, what types of items were typical, and which stitches and techniques were preferred to gain additional information from what was found in the interviews. An amendment to the original research request was sent to the Human Research Subjects Committee at 
Colorado State University. Upon approval on March 7, 2011 the researcher sent a short e-mail thanking the participants and asking them to take a few minutes to fill out the attached survey.

\section{Data Analysis}

\section{Interviews}

Interviews were taped using a digital voice recorder and a transcript was generated by the researcher for each participant. The interviews were labeled with the assigned code of the participant. Data gathered from the participant interviews were analyzed using constant comparison method which was defined as a systematic method of comparing concepts against one another to see how they relate to a similar phenomenon (Strauss \& Corbin, 1990). It involved three types of coding that helped to identify, examine, and then build the data into understandable units.

The first step in the constant comparison method was concept identification, which required that the researcher identify data that focused on similar phenomenon and named concepts in a process called open coding (Denzin \& Lincoln, 2000, p. 516). Concepts were then further grouped into larger and more abstract units called categories (Strauss \& Corbin, 1990). The categories were recorded into a code book which had specific instructions and criteria as to how they applied to each category (Gibson \& Brown, 2009).

The study then progressed to the second stage of coding, axial coding, which made connections between the categories and their subcategories (Denzin \& Lincoln, 2000, p. 516). As the data was fractured during the open coding process, it was then rebuilt in the axial coding stage. The goal of this stage was to create larger, more 
abstract, and encompassing categories out of the data that were sensitive to the social context and conditions that gave rise to the original text (Strauss \& Corbin, 1990). This was accomplished by looking for linkages among the categories and subcategories and then examining the patterns created within those linkages (Strauss \& Corbin, 1990).

Selective coding was the third and final step in the coding process of constant comparison method (Denzin \& Lincoln, 2000). This process involved taking the core categories and then comparing them to the other categories to validate the relationships, and determine if further development was needed within a category (Strauss \& Corbin, 1990, p. 116).

Through this process the researcher identified emergent themes in an inductive process (Gibson \& Brown, 2009). The researcher then compared the themes to existing theory in a deductive process (Gibson \& Brown, 2009). In order to understand the incentives of the participants, the emergent themes were compared to Uses and Gratifications Theory and the categories of motivations for participation as suggested by Katz et al (1974).

\section{Knitted Artifacts}

The Knitted Artifact Instruments were filled out and analyzed according to the methods suggested by Smith (1985). All the observable information portions of the instruments were analyzed by the researcher in an open coding process that is part of content analysis method. The photographs of the artifacts were used to facilitate this process. Content analysis method operates on the principle that all artifacts are infused with cultural meanings and can serve as evidence for the specific culture that produced 
them (Fleming, 1986). Comparable data from www.ravelry.com was also considered to add cultural meanings.

The researcher compared the data to supplementary knitting literature. Themes that pertained to the artifacts were taken from the observable, comparative, and supplementary sections of data. The observations of the researcher during coding were also examined for themes. The analysis sought to find links among the values and beliefs of the knitters' culture and the actual items that were produced (Fleming, 1986).

\section{Post Interview Survey}

The Post Interview Participant Survey (PIPS) was analyzed using Statistical Package for the Social Sciences (SPSS). Eleven questions from the survey were examined and answers for both the demographic and knitting preference data were entered into the program. Frequencies and descriptive analyses were used to interpret the data.

\section{Validity and Reliability}

\section{Validity}

Triangulation in qualitative research refers to investigating the same concept or phenomenon with different methods and from different viewpoints to validate the research study (Gibson \& Brown, 2009). It is of utmost importance to triangulate the data gathered because it establishes validity or trustworthiness throughout the different types of data by showing commonalities across various types of sources (Gibson \& Brown, 2009).

The researcher utilized multiple types of sources to triangulate and ensure the validity of the data. The first source was the data gathered from the interviews with the 
participants. The second source came from the Knitted Artifact Instrument. A third data set was gathered for the comparative data section of the Knitted Artifact Instrument which was obtained from www.ravelry.com. The fourth set of data came from the supplementary data section of the Knitted Artifact Instrument; this information came from books and dictionaries on the subject of knitting. The final data source came from the Post Interview Participant Survey.

\title{
Reliability
}

In qualitative research it is important to ensure reliability of the data, meaning that the researcher's approach to interpreting data is consistent with other researchers' interpretations of the same information (Creswell, 2009). The data utilized in this study were checked for reliability to ensure that the findings of this research study were valid.

The researcher interviewed the first participant, created a transcript, and analyzed the data using constant comparison method. The codes that resulted from this first set of data were recorded into a code book. A second coder with a design and fiber arts background was then asked to look at the same data to establish intercoder reliability which has members of the same academic field check the analytic categories, conclusions, and interpretations of the researcher (Tashakkori \& Teddlie, 1998). These two sets of codes were cross-checked to ensure that the researcher was interpreting the results reliably. The following equation was used to calculate the consistency between coders.

\author{
Agreements - Disagreements $\times 100=$ Reliability \\ Agreements
}


A consistency of at least $80 \%$ is expected to prove intercoder reliability in qualitative research and was anticipated between the two sets of codes (Creswell, 2009). A percentage of $80.09 \%$ was achieved by the researcher and secondary coder so no adjustments were made to the codes. 


\section{CHAPTER FOUR}

\section{RESULTS}

Three sources of data (post-interview participant surveys, semi-structured interviews, and knitted artifacts) were analyzed in an effort to detail the relationship between knitting and young women. Each of these sources provided valuable information about young, female knitters and their specific culture. In order to best disseminate the information gathered results are offered starting with participant demographic data, knitting frequencies and preferences. Next, the major themes and subthemes from the interview data will be highlighted. Finally, the material culture of the knitted artifacts produced by the participants will be examined.

\section{Participant Demographics}

The Post Interview Participant Surveys (PIPS) was administered via e-mail after the initial analysis of the interview and material culture data. Fourteen of the knitters completed the survey and one woman declined to participate. Data gathered from the PIPS were analyzed using Statistical Package for the Social Sciences (SPSS). Demographic and knitting preference data gathered from the PIPS were analyzed using frequencies and descriptive statistics. 
The demographics of the participant sample are shown in Table 4.1. The participants were between the ages of 20 and 29 years old, the majority were either 22 (29\%) or 29 (29\%). In terms of knitting experience, six (42\%) of the women had three or less years of knitting practice, while eight (58\%) had over five years. All of the women surveyed had at least some college education and seven (50\%) had earned a bachelor's degree. The average income for the participants varied as well, the majority (79\%) of the women made under $\$ 25,000$ per year. The relatively low earnings most likely resulted from the youth of the majority of participants and the fact that many were still college students. 
Table 4.1

Demographics of Participant Sample of Young Female Knitters

\begin{tabular}{|c|c|c|c|}
\hline \multicolumn{4}{|l|}{ Participants $(n=14)$} \\
\hline \multicolumn{2}{|l|}{ Current Age ( $n=14)$} & \multicolumn{2}{|c|}{ Years of Knitting Experience $(n=14)$} \\
\hline 18 & $0 \%(n=0)$ & $<1$ & $0 \%(n=0)$ \\
\hline 19 & $0 \%(n=0)$ & 1 & $21 \%(n=3)$ \\
\hline 20 & $7 \%(n=1)$ & 2 & $14 \%(n=2)$ \\
\hline 21 & $14 \%(n=2)$ & 3 & $7 \%(n=1)$ \\
\hline 22 & $29 \%(n=4)$ & 4 & $0 \%(n=0)$ \\
\hline 23 & $0 \%(n=0)$ & 5 & $7 \%(n=1)$ \\
\hline 24 & $0 \%(n=0)$ & 6 & $0 \%(n=0)$ \\
\hline 25 & $0 \%(n=0)$ & 7 & $14 \%(n=2)$ \\
\hline 26 & $7 \%(n=1)$ & 8 & $7 \%(n=1)$ \\
\hline 27 & $7 \%(n=1)$ & 9 & $0 \%(n=0)$ \\
\hline 28 & $7 \%(n=1)$ & $>10$ & $29 \%(n=4)$ \\
\hline 29 & $29 \%(n=4)$ & & \\
\hline \multicolumn{2}{|l|}{ Education $(n=14)$} & \multicolumn{2}{|l|}{ Income $(n=14)$} \\
\hline Some College & $43 \%(n=6)$ & $<\$ 10,000$ & $36 \%(n=5)$ \\
\hline Bachelor's Degree & $50 \%(n=7)$ & $\$ 10,000-\$ 25,000$ & $43 \%(n=6)$ \\
\hline \multirow[t]{4}{*}{ Master’s Degree } & $7 \%(n=1)$ & $\$ 25,001-\$ 40,000$ & $14 \%(n=2)$ \\
\hline & & $\$ 40,001-\$ 65,000$ & $7 \%(n=1)$ \\
\hline & & $\$ 65,001-\$ 80,000$ & $0 \%(n=0)$ \\
\hline & & $>\$ 80,001$ & $0 \%(n=0)$ \\
\hline
\end{tabular}

The PIPS also measured knitting involvement and preferences which are shown in

Table 4.2. The knitters ranked themselves in terms of perceived skill level, half of the participants (50\%) identified themselves as intermediates. Of the remaining knitters, four (29\%) of the women considered themselves to be advanced and only three (21\%) were beginners.

There was a distinction made as to whether the knitters were process or product knitters. Process knitters were primarily interested in the physical act of knitting and less 
in the products that resulted, whereas product knitters were concerned with the final projects and cared less for actually knitting. There was a fairly even split between the sample, eight (57\%) women identified themselves as process knitters and the other six (43\%) felt they were product knitters.

The PIPS gauged the amount of investment in materials and knitting preferences as well. The participants spent anywhere between less than one hour to nine hours knitting in an average week and were fairly evenly distributed between the time categories. The women also disclosed the average investment made in purchasing knitting materials made per month. Four (29\%) women spent less than $\$ 10$ a month on supplies, seven (50\%) paid an average of $\$ 10$ to $\$ 25$, and only three (21\%) spent over \$25 a month on knitting materials.

The knitters were asked to identify what types of projects they had completed within the last year, the most frequently made items were scarves which twelve (86\%) of the participants. Gifts and hats were also popular projects; both were made by nine (64\%) knitters each category. Participants were asked to describe the types of yarn purchases they had made in the past year, wool had been purchased by every participant (100\%). Acrylic was purchased by twelve (86\%) women and cotton was obtained by ten (71\%). Interestingly, half (50\%) of the women had also bought eco-friendly yarns over the past year.

Finally, the knitters detailed the techniques they preferred to use in their projects. Knitting in the round was preferred over flat knit with nine votes (64\%). Cables were also highlighted as a favorite technique by nine (64\%) women. Color work was the least popular technique with only five (36\%) women having favored to use this method. 
Table 4.2

Knitting Preferences of Participant Sample of Young Female Knitters

\begin{tabular}{|c|c|c|c|}
\hline \multicolumn{2}{|l|}{ Participants $(n=14)$} & & \\
\hline $\begin{array}{l}\text { Skill Level }(n=14) \\
\text { Beginner } \\
\text { Intermediate } \\
\text { Advanced }\end{array}$ & $\begin{array}{l}21 \%(n=3) \\
50 \%(n=7) \\
29 \%(n=4)\end{array}$ & $\begin{array}{l}\text { Type of Knitter } \\
\text { Product } \\
\text { Process }\end{array}$ & $\begin{array}{l}57 \%(n=8) \\
43 \%(n=6)\end{array}$ \\
\hline $\begin{array}{l}\text { Average Time Spe } \\
\text { Week }(n=14) \\
<1 \mathrm{hr} \\
1-3 \mathrm{hrs} \\
3-5 \mathrm{hrs} \\
5-7 \mathrm{hrs} \\
7-9 \mathrm{hrs} \\
>9 \mathrm{hrs}\end{array}$ & $\begin{array}{l}\text { ting in a } \\
\begin{array}{r}14 \%(n=2) \\
21 \%(n=3) \\
21 \%(n=3) \\
29 \%(n=4) \\
14 \%(n=2) \\
0 \%(n=0)\end{array}\end{array}$ & $\begin{array}{l}\text { Average Monthl } \\
\text { Supplies }(n=14) \\
<\$ 10 \\
\$ 10-\$ 25 \\
\$ 26-\$ 40 \\
\$ 41-\$ 65 \\
\$ 65-\$ 80 \\
>\$ 80\end{array}$ & 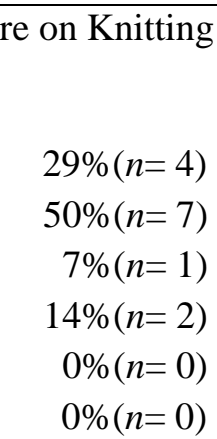 \\
\hline $\begin{array}{l}\text { Types of Knitted It } \\
\text { the Past Year ( } n=1 \\
\text { Sweaters } \\
\text { Other Garments } \\
\text { Gifts } \\
\text { Toys or } \\
\text { Scarves } \\
\text { Hats } \\
\text { Fingerless Gloves } \\
\text { Blankets } \\
\text { Other Gloves, } \\
\text { Mittens }\end{array}$ & 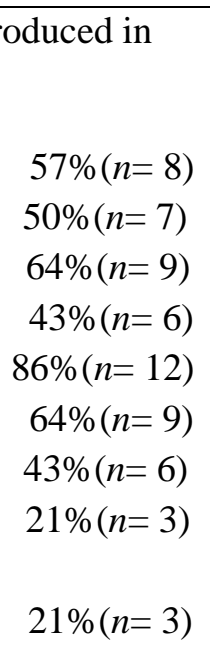 & $\begin{array}{l}\text { Types of Yarn P } \\
(n=14) \\
\text { Acrylic } \\
\text { Cotton } \\
\text { Silk } \\
\text { Wool } \\
\text { Mohair } \\
\text { Alpaca } \\
\text { Novelty Blends } \\
\text { Hand Spun } \\
\text { Hand Dyed } \\
\text { Eco-friendly }\end{array}$ & 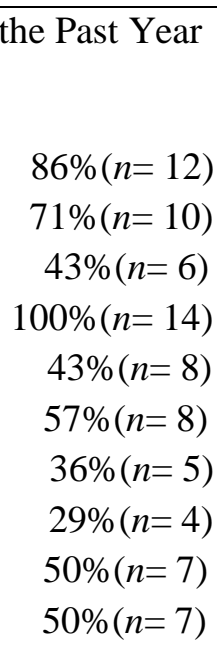 \\
\hline $\begin{array}{l}\text { Preferred Techniqu } \\
\text { Cables } \\
\text { Color Work } \\
\text { Lace } \\
\text { Knitting in } \\
\text { the Round } \\
\text { Flat Knit }\end{array}$ & $\begin{array}{l}14) \\
64 \%(n=9) \\
36 \%(n=5) \\
43 \%(n=6) \\
64 \%(n=9) \\
57 \%(n=8)\end{array}$ & & \\
\hline
\end{tabular}




\section{Interview Data}

Upon analysis of the interview data three major themes emerged: incentives, barriers, and outcomes. The three main themes were comprised of thirteen subthemes: creativity, multi-tasking, financial, alterations, information sources, social aspects, negative reactions, mistakes, expense, positive reactions, products, confidence, and relaxation. The Taxonomy of Participation in Knitting, was developed to visually categorize the themes and subthemes within the data it is displayed as Figure 4.1. 
Figure 4.1

Taxonomy of Participation in Knitting

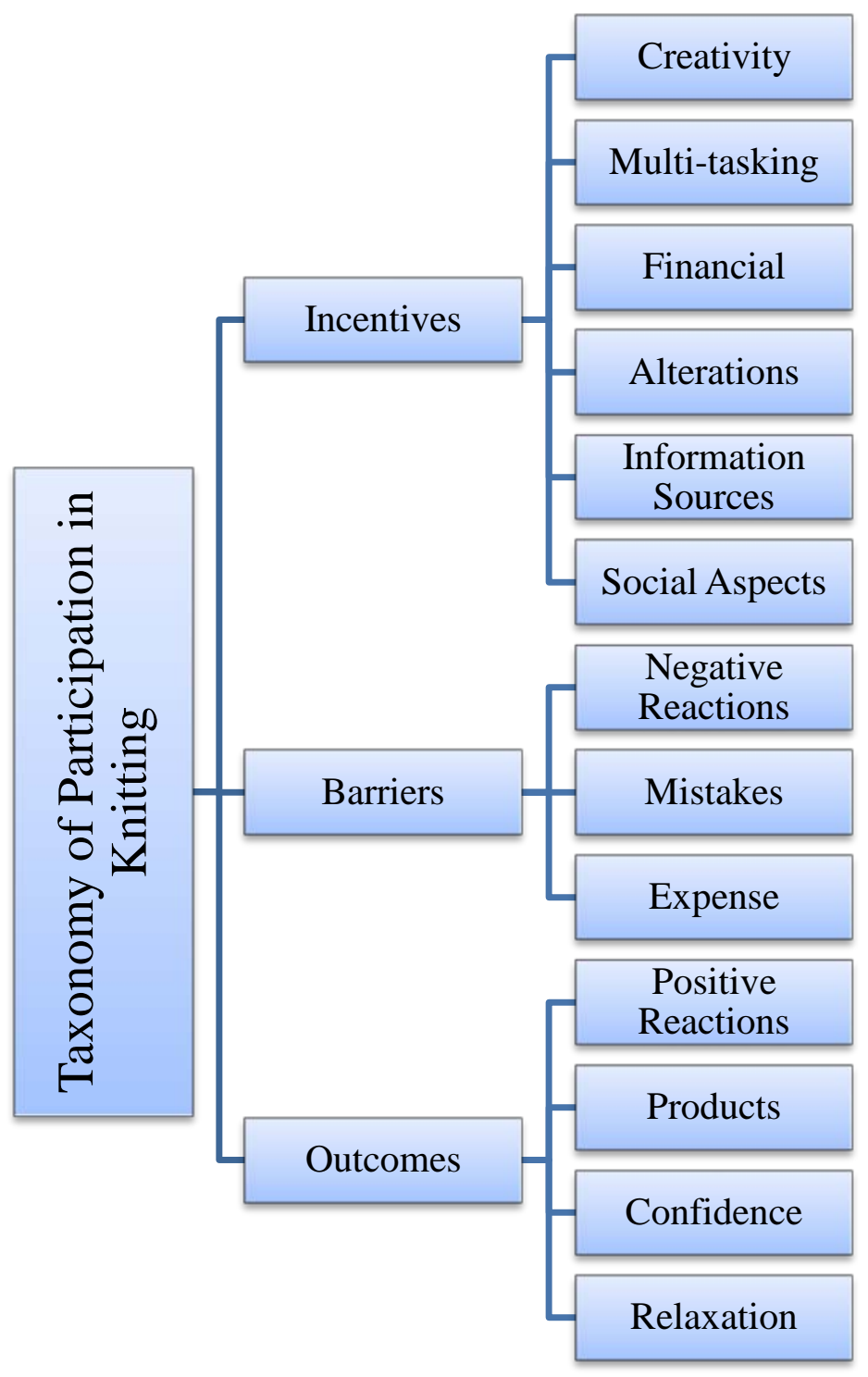

Incentives

The theme of incentives examined the various supportive elements for knitting. Motivations for participating in knitting by young women were also emphasized within 
the theme. The incentives theme was comprised of six subthemes: creativity, multitasking, financial, alterations, information sources, and social aspects.

\section{Creativity}

Knitting allowed the participants a creative opportunity which otherwise may not have been part of their daily lives. Knitting, "gives me a creative outlet...I work at an insurance agent's office selling insurance, which is about as least creative as you can possible be” (participant 1). The participants also felt knitting had a general lack of rules, which allowed them to be creative with the construction of their projects.

Some of the knitters were described as very creative and even intuitive with their process. These individuals were able to "eyeball" size and shape of patterns. Oftentimes "intuitive" knitters would modify the size of an item while working on a project. For instance, “I don’t like to follow a pattern completely like if you have a sock I would much rather start at the toe and then keep adjusting it as I need to" (participant 5). Many of the intuitive knitters did not use patterns, because they felt patterns were too limiting or they found stringent counting required by some patterns to be annoying.

Inspiration for knitting projects came from a variety of sources. Physical needs were discussed as a source of inspiration for knitting both garments and accessories in this instance one participant stated, "this winter I wanted something stylish and warm" (participant 13). Knitters in this sample displayed cases of synthesizing creative cognitive processes and inspirational fashion trends, for example, "what could I do to make a unique earflap hat ...I like to do something that's popular so something that everyone is making” (participant 10). Another area for inspiration was popular culture, items 
inspired by series such as Twilight and Harry Potter spurred knitters to create garments featured in the films and books.

The knitters were excited to create items they perceived as distinctive or individualized. One woman illustrated this idea:

And then with this one I just feel so happy when I have it on because like I said no one else has, it's all mine, no one else has it. Even if someone were to do the exact same pattern, even in the exact same yarn it would still be completely different (participant 9).

The sense of creating a one-of-a-kind translated into gifts made by the knitters as well. The perceived uniqueness or custom-made nature of a gift was viewed as being significantly better than store-bought, one knitter claimed, “I feel like it’s more personalized like the gifts, it’s better than like a gift card to Target, it's like I spent 10 hours on this and I made this specifically for you” (participant 11).

\section{Multi-tasking}

The ability to multi-task was commented on by all participants, who enjoyed knitting while doing any number of activities such as waiting or sitting still. One woman explained, "I just went to Disney World in December for a trip and I brought knitting because that's what I do; there’s long lines” (participant 9). Participants were happy to pull out their latest project and knit a few rows while in line at the post office, waiting for class to start at the gym, and even while sitting in the car. A number of reasons were cited for knitting in public, but most had to do with increasing tolerance and allowing for a quick relaxation session. For instance:

When I'm in line at the DMV and it's going to be 20 minutes, those are the times when I'm like ahhh, my patience would be so much better right now if I had something to do with my hands (participant 4). 
A majority of the participants self-identified as "fidgety" people who would bounce around, pick at their nails, or be unfocused during their daily routines without something to do with their hands. This was clarified by one participant, "I'm kind of fidgety and I don’t know if this is just a characteristic in knitters, but you know sometimes I feel like I have a lot of nervous energy and by knitting it sort of calms me down” (participant 13). The women claimed knitting allowed them to keep their restless hands busy, which enabled them to really focus on the other task at hand. Because of the ensuing calm nearly all the women enjoyed knitting which completely stationary and needing to be attentive, such as watching television or listening to classroom lectures. In fact, the knitters who worked during classes claimed it improved their absorption of the knowledge along with their ability to pay attention.

\section{Financial}

The cost of knitting was fairly significant for the participants, especially those who were producing large projects. The women worked in a number of ways to counteract the costs associated with knitting. Some of the participants were quite skilled in locating materials at little or no cost. These knitters shopped thrift stores for materials and even recycled yarn from previous projects. One woman explained how she kept her costs low, she stated, "I usually use yarn that was a gift, or these were from a thrift store, or scrap bin fabrics I'm an art student so there's always stuff in the scrap bin” (participant 8).

The participants were oftentimes experienced coupon shoppers who watched yarn sales closely, especially for online stores. Yarn retailing websites were especially enjoyed by participants, for example, “I really like Knit Picks, it has pretty yarn...and 
they also run pretty good sales, like after Thanksgiving they had all their yarn on sale” (participant 1). When the participants were able to find yarn on sale they often engaged in "stash" building by purchasing yarn for future unspecified projects. Possessing a stash of yarn allowed knitters to minimize costs. However, some women expressed that stashing yarn may not be advantageous as they were unable to predict future project needs and there may not be sufficient yardage within their stash to complete projects.

\section{Alterations}

Alterations to patterns served as one of the main challenges to the participants. Adjustments varied in complexity from simply changing the type of stitch or yarn to completely resizing a knitted garment. Some of the less skilled knitters were intimidated by trying to alter, one beginner stated, “I'm kind of nervous about making a sweater because I know I'll come across where I'll have to change some things” (participant 7). Modifying larger pieces made a few knitters especially hesitant, because if the alterations were not correct they would have to take out a large portion of their knitting.

When altering a pattern it often became necessary to plan for the project.

Depending on the complexity of the change, some participants would use graph paper to map out stitches to better predict the outcome. For instance, "where it gets particularly hard is when you're doing lace or something with more complicated textures, something like that you have to do a little more mapping” (participant 2). The process of modifying a written pattern was very dependent on math and counting and many knitters found it to be difficult, but fun. Surprisingly, one knitter was a mathematician who aided many of her knitting friends with alterations. She declared, "people ask me for help on their patterns all the time” (participant 3). 
Alterations were ultimately embraced by many of the participants. For knitters the motivator for many alterations, "was trying to expand what I know, so trying new, different stitches and patterns” (participant 12). Some of the participants described looking for projects that offered a new challenge, so with every project they would become more knowledgeable and improve their knitting skills.

The participants were also skilled in terms of choosing yarns for their projects. They understood how certain fibers would ether benefit or detract from their project goals, for example, "alpaca's really warm so I decided that I'd go ahead and try a scarf” (participant 10). Fiber consideration was one of the primary decisions made before beginning a project. The participants conversed about how they chose their yarn; many demonstrated a complex and detailed understanding of fibers. One woman demonstrated her fiber knowledge in regards to decision making process in her explanation, "I figured that care wise and storage wise that cotton would be easier than a lot of other fabrics, or fibers” (participant 4).

\section{Information Sources}

A variety of information sources were available to the knitters. Nearly all of the participants were either members of, or at least aware of www.ravelry.com. The website was viewed as a staple in the knitting world by many of the women, for instance, "it's an awesome community and I honestly don’t know how people knit before it” (participant 3). The website's features included discussion boards, groups, contests, yarn for purchase, help, and a large pattern library with many free patterns. Upon describing the pattern offerings one woman stated, "holy cow, I was floored the first time I went on there, like there’s that many patterns for knitting” (participant 9). 
There were a host of other websites the knitters visited to find information, help, tips, and patterns for projects. Videos on the Internet were helpful, "if I needed to look something up in a pattern, especially for this sort of flowery lattice, I just went to YouTube and Googled SSK2” (participant 15). A couple women were enamored with websites such as www.knitty.com, which featured articles on techniques as well as free patterns. A few of the knitters were particularly interested in certain brands, for example, "I like Lion Brand yarn a lot and I go to their website and they have a lot of patterns and I kind of take inspiration from there" (participant 11).

Books and magazines offered another alternative for knitters to gain information and patterns. One knitter preferred to have a book because it had an extended glossary of stitches within the pages. She explained:

I've tried some free knitting patterns from online, but I don't really like them because they don't have a reference in the front or something to help me figure out what some of the short hand or abbreviated parts are (participant 12).

An unexpected source was the library, one knitter commented, "I love the library, love the library, they have great, a really good number of books” (participant 9). It was commonly mentioned by participants as a good place to obtain knitting information. Access to Prospector, an extended library system, allowed a few knitters entry to a larger number of libraries in the region and thus even more knitting books. By checking out books of patterns the knitters could either complete a project prior to the due date or they were able to search for favorite projects and make photocopies for later.

Retail establishments catering to knitters were also patronized by participants in search of patterns and information. Some had glowing reviews, "it's good, (the owner) 
was super helpful she's like my age and doesn't mind that I'm always hanging out around there” (participant 7). While others were not preferred, "I go to knitting shops especially like the (local yarn shop) because they have tons of books, but I don't want to buy anything there because they're kind of snoody" (participant 7). A couple of the younger knitters explained that they felt staff in certain yarn stores looked down on their smaller projects. Knitters were also concerned with being able to get help without having to buy materials in the same trip, which was not allowed at certain shops.

\section{Social Aspects}

Nearly all of the participants were part of a knitting circle and attended "Knit Nights,” which were public gatherings of a knitting circle, regularly. The women had various reasons for participating in the social groups, but overall they enjoyed the company of other knitters who understood their hobby and shared the same passion. Making friends was cited as one of the best parts of the entire knitting culture, one woman claimed, “it’s opened a lot of doors for me socially, I think, I’ve met a lot of good friends through knitting” (participant 3). The shared interest and excitement for knitting was continuously mentioned, for example, "it’s just a really nice relaxed group of people who all have the same dorky interest in knitting that no one else in my life understands" (participant 4).

Engagement with other knitters created an opportunity for many of the participants to learn new skills. They were able to bring projects to knitting friends and events like "Knit Night" to get advice on how to fix mistakes. In addition, other knitters often provided design feedback for participants such as yarn and alteration suggestions. Teaching was another way for the knitters to cement their skills and to pass along 
knowledge. A couple of the newer knitters were eager to teach, one participant remarked, "if there's someone who wants to learn something I want to teach them that because that makes me better” (participant 3).

Specific pattern and project ideas were regularly gained from other knitters. One knitter discussed how she might obtain patterns from other knitters, she stated, "if I meet a group of ladies out doing a "Knit Night" then at least one person is wearing something that they made themselves, so if I like that pattern I ask them what it is” (participant 1). Others knitters created a social support system which allowed for the advancement of knitters. By sharing patterns, the women encouraged their friends to continue with the craft and to develop new skills.

The larger knitting community has created a number of different events to promote knitting. A couple of the participants were actively engaged in "swaps" via www.ravelry.com. Swaps were elaborate knitting gift exchanges conducted over the social networking website. To participate a knitter would sign up for a particular theme, which ranged from popular culture topics such as "Tim Burton movies" to nature themes like "ocean and sea." Once registered, the knitters were paired up in a round robin fashion by the swap's organizers. The knitters then had to research the likes and wants of the person they had been paired with to understand what type of present that individual would like to receive. Knitters were given two months to knit one large item (though other techniques such as sewing were also allowed, knitting was the predominate) for their recipient. At the end of the swap the knitters prepared a package containing the large knitted item, a medium sized item (typically yarn), and a smaller item like chocolates to send along with the item. 
Upon receiving their packages recipients were expected to post a thank you on the swap's discussion board detailing what they had received. Other members of the swap group also posted compliments and messages on the discussion board. One avid swapper explained, "I love having someone post an awesome thank you to me in the thank you thread" (participant 4). These posts and compliments affirmed that the knitters had done a good job and often pushed them to participate in more swaps.

Other events held on www.ravelry.com included Ravolympics, during the Olympics celebration knitters formed teams and participated in various events and knit-alongs in which a group all knit the same pattern. Two participants had even created their own events, one woman had belonged to a knitting book club. She explained:

I did a book group that we called ourselves the Knitting Readers and we would have a book group where you choose a title every month and then get together once a month and talk about it, but we would knit while we talked about it. (participant 1)

Another woman had held an alternative Superbowl party, a "Sue Pearl Bowl” party. Stephanie Pearl-McPhee is a well-known figure in the knitting world and the party was for celebrating knitting instead of watching the football game.

\section{Barriers}

Barriers to knitting represented the negative aspects of the knitting process. There were obstacles to knitting from other people as well as from the knitters themselves. The barriers theme was comprised of three sub themes: negative reactions, mistakes, and expense. 


\section{Negative Reactions}

Participants had encountered a number of different negative reactions to their knitting. A large portion of the knitters had been openly teased by strangers, friends, and family about their hobby. One woman found that her family in particular could not understand why she was passionate about knitting, she reflected, “they don’t necessarily devalue the things that I produce, but they don't value the craft of knitting” (participant 4).

The sense that the "craft of knitting” or process involved in creating a product was devalued was experienced by many of the participants who sometimes felt as if they were being watched while knitting in public spaces. They reported that observers were typically nice and generally interested, but there were occasional negative comments that were hurtful to the women. A couple knitters reported being taunted about the items they were making, for instance, “I’ve certainly been teased about it, people either see me knitting a sock or they find out that I'm wearing socks that I knitted and they ask, well can’t you just go to Wal-Mart and get a package of ten?” (participant 1). Other observers harassed the knitters about being old ladies; one woman remarked, "it’s funny that people just assume that like grandmas or old ladies knit” (participant 6).

Knitting, especially when done in public, was not well understood and often caused confusion amongst observers. For instance, participants stated that many people confused knitting and crochet. While a few participants felt empowered by the “rareness” of their hobby and general lack of understanding, others were annoyed by the confusion of knitting with crochet. The annoyance may have resulted from the fact that many of the knitters did not view crochet in the most positive light. One woman 
explained why she favored knitting, she claimed, "I like the look of knitting more than crocheting just because crocheting kind of reminds me of old lady afghans and weird dollies and stuff” (participant 7).

Participants also commented that their hobby was prone to oversimplification by members of the public. The generalization of their knitting projects was irritating to many of the participants, case in point, 'they're like, 'oh is that knit, purl, knit, purl' and I'll be doing a fancy lace stitch” (participant 8). Frustration over public comments caused a couple women to avoid public knitting.

Unappreciative gift recipients were damaging to participants as well. A majority of the women expressed that the worst thing for a knitter was to have a gift recipient who did not value their present. When gifts are not appreciated, "it makes me want to knit for me more, make something really nice for myself” (participant 2). Unappreciative gift recipients were also removed from the list of people the knitters were willing to make items for.

\section{Mistakes}

Mistakes were a typical occurrence for many of the participants. Sometimes they resulted from incorrect technique or general lack of know-how. For example one knitter was making a pair of fingerless gloves; she did not known the correct bind off method which caused a fit mistake. She admitted, “I don’t think that that's going to suit the purpose, unless I learn how to do the right bind off on this one and then just wear it on my left hand, I can't move my hand in them” (participant 7). Errors were also made on the design end of the process and some participants discussed how they had made 
mistakes with the color choice, fit, or feel of their projects. One knitter explained a poor design decision she had made:

...this was a decision that I regretted and then half of it I made with this really cool multi colored yarn it was like green, and blue and white, then I ran out and I decided to just keep going with just like lime green color that had been pulled out of the mix of colors. And I thought that it'll just be behind the neck, but I hated it after (participant 6).

Other projects were rather stressful to the knitters for a variety of reasons and were sometimes abandoned by participants. Boredom with parts of the knitting process would lead to desertion, one knitter mentioned, "it was so tedious and time consuming and there was not enough progress on it to be satisfying and so I gave that up” (participant 3). Sometimes abandoned projects would become interesting to knitters in the future, so they were often stored or as one participant put it they became "dormant" projects. Nearly all the participants had active projects and dormant projects in their knitting bags. Projects that were considered to be complete failures were called "whips" and were often disposed of or "frogged" unraveled back to yarn. Surprisingly, participants had an overall blasé attitude towards their mistakes, for instance, "so I have as many whips as finished objects at any given point in my life” (participant 4). The indifferent attitude towards whips was also carried through by a number of participants who were happy to embrace mistakes within their knitting.

However, others were quite distressed by mistakes. A few women described a sense of unhappiness with aspects of their final projects, for example, "like my fingerless gloves, I totally need to repair them and I kind of feel self-conscious about it even though it's cold, you have to wear them, and I kind of like want to hide them a little bit or hide 
certain parts of them” (participant 5). Sometimes knitters even altered their project or pattern choices in order to avoid errors.

\section{Expense}

The final barrier to knitting came from the actual expense of the materials needed to participate. Cost was a major factor for the participants, many of whom were students or living on a smaller income. Participants spoke about wanting to have quality fibers, one knitter admitted, “I’ve always been kind of a fiber snob, I really only want use like the good stuff, it usually ends up being the best that I can afford” (participant 2). A portion of the knitters described how they were simply unable to afford the higher level of materials available in the marketplace, which limited their ability to make projects.

The expense of a proposed project was stressed as an important consideration to be made in the planning stages of a knitting project. When the preparation was not done correctly it could become quite costly for the participants to finish a project. Without proper foresight knitters may have had to purchase additional supplies this was illustrated by a knitter’s scarf project, “I needed like an extra ball of yarn then I thought so the scarf turned out to be like a \$50 scarf, because I didn’t calculate it right” (participant 7). A couple of the knitters decided to avoid larger projects like garments because of the high yardage requirements and the expense they would incur.

\section{Outcomes}

Outcomes to knitting represented the end results of the knitting process. The beneficial results of knitting oftentimes encouraged knitters to continue in the craft. The themes of outcomes encompassed four subthemes: positive reactions, products, confidence, and relaxation. 


\section{Positive Reactions}

Knitters receive a number of positive reactions to both the final products and the actual process of knitting. Compliments from others fostered a sense of pride in many of the knitters, one participant remarked, "I like when I get compliments and I can say that I made it" (participant 3). Being able to say "I made it" was so enjoyed that some of the women admitted to showing off finished projects in order to get people to ask if they had made the item. One knitter explained:

It's like when you get a new dress and you just walk around waiting for someone to ask you about it so you can brag...a little added satisfaction to finding that dress you really love is that you got to make it yourself. (participant 1)

Recipients of hand knit gifts provided positive feedback to the women by appreciating and enjoying the knits made for them. Participants were excited to see the knitted gifts they had created worn by recipients and many stated that it drove them to produce even more projects. Positive feelings about gifting were displayed, for example, "I think that giving stuff to people shows how much you care, it not only gives you a sense of satisfaction when they like it and use it” (participant 13). One knitter remarked, "sometimes you get people who even if they really appreciate it they put them away for special or for nice and these things are made to be used” (participant 2). The overall consensus was that knitters wanted the gifts they had created to be worn by the recipients on a frequent basis.

The reinforcement offered by others was very important to the participants. The knitters highlighted how friends and family members supported and encouraged them. In addition, the backing and approval of other knitters bolstered a feeling of 
accomplishment. These positive opinions often led the participants to try new patterns and learn more skills, for example, "when you get that feedback on your work you think I tried something new, I stepped out of the box on this one and I'm going to try something even more difficult on my next one” (participant 3).

\section{Products}

The participants were involved in creating a number of different types of products. Interestingly, nearly all of the participants had either made or were making fingerless gloves, which appeared to be one of the most sought after projects. Fingerless gloves were actually the motivator for one woman to become a knitter, she remembered, "the girl that I worked with who was knitting was making fingerless gloves and I wanted to make those” (participant 1).

Accessories in general were the predominate type of product created by the knitters. Accessories were viewed as more popular projects, "I think probably because they're quick and I like instant gratification” (participant 12). In addition to being quick, accessories were small enough to be portable, which was another major point of interest. A significant number of the participants enjoyed knitting in a variety of venues and claimed to always have a portable project with them.

Larger projects, such as blankets and garments were not nearly as popular with the knitters. The lack of portability was a definite deterrent, one participant remarked, “right now I have this sweater that I'm knitting ... and it's like a pretty big chunk of fabric so it's too big to carry with me” (participant 8). A number of the knitters were worried about the cost of materials to make garments and were daunted by trying to understand how to fit garments correctly as well. The knitters who felt unprepared to 
work the stitches required for a garment often decided to avoid larger projects until they gained more knitting experience. Seaming was also mentioned as an intimidating part of garment construction. For instance:

I haven't done a lot of garments because the stitching all together kind of scares me and it requires a lot of yarn and I don't have that much yarn in one color or style yet to do big garments (participant 9).

Creating functional and useful products was very important to knitters. They were inspired, "from needs like this winter I wanted something stylish and warm" (participant 13). Toys and novelty items were occasionally made, but the knitters described them as un-useful. As a whole, they were less interested in the toys, one woman admitted, "I do get a little bit of pleasure, but not maybe as much some people do because it’s a ghost (toy) what do you use a ghost for?” (participant 5).

Gifts were a huge motivator for the participants; all but one had given some of their knitted projects away. A majority of the knitters felt this was the most rewarding part of the knitting process. The women also talked about the advantage of knitted gifts, one knitter explained, "I think the best gift that you can give is something knitted" (Participant 3). The participants were happy to give knits, for instance, "it’s a lot different; to go out and buy something is really simple, but to really understand what someone likes and being able to use your own hands and make it” (participant 10). Gifts were also an avenue for one knitter to get rid of various knitted items she had created, but was not interested in using.

Another type of giving supported by the knitters was knitivism, which was practiced by three of the participants. The knitters only took part in charity based knitivism and were not involved in any environmental or political knitting ventures. One 
woman explained her knitivism involvement, "on Tuesdays I get together with these ladies, these older ladies from my church, so we get together and we knit these hats and blankets for the Alpha Center; it makes it more worthwhile for me” (participant 10). Other projects included Christmas gifts for a local women’s shelter and pickline covers, which are covers for IVs that distributing chemotherapy medicines, for cancer patients at a local hospital.

\section{Confidence}

The majority of the participants felt very confident about their projects and skills as knitters. They were proud of their abilities and some felt they had a valued talent, for example, “I love to knit; it makes me feel...I don’t know it makes me feel unique and special” (participant 9). Another knitter commented, “I’m super happy, proud of myself, and excited about what I'm doing, that's really the pay off" (participant 3). In addition, many of the knitters were extremely satisfied with the projects they had completed. The women were also able to post their work on www.ravelry.com or take it to "Knit Nights" to show other knitters who provided positive feedback.

Another confidence building aspect of knitting was gaining knowledge. Nearly all of the participants discussed how hard knitting was for them originally as they did not understand stitches and how patterns fit together, as they practiced and gained knowledge they were able to appreciate how the various elements of knitting worked together to make patterns and projects. Understanding how knitting works, “makes me feel like I know what I'm doing” (participant 14). The participants felt increasingly confident in their skills as they learned how knitting worked and could anticipate results of patterns and stitches. 
The knitters also reported feeling happy because they were able to be productive. The ability to simultaneously knit while doing other tasks made knitters feel industrious, one woman decided, “you get a product out of it...I guess it’s better to watch TV and knit at the same time” (participant 6). A few were happy to knit while being with friends and family members, because they were able to socialize while getting their projects accomplished. Knitting while socializing also increased mental focus, for instance, "that's one of those things that I absolutely love about knitting is because it makes anything social and completely not like I'm being 'ah, I want to be doing something else' because I am doing something” (participant 4).

Aside from feeling productive, some of the knitters stated that they were becoming less dependent on consuming. One woman discussed how knitting is, "sort of self sustaining and being like well I don't need to go buy those things because I can make it myself" (participant 13). The knitters who did feel self-sustained through knitting were excited to take a step back from consuming. Others described how knitting reminded them of the past when everyone made their own garments from necessity.

\section{Relaxation}

The stress relief that resulted from the physical process of knitting was essential to the knitters. It allowed them to unwind and one mentioned it was her personal form of meditation. The stress relief benefits were highly important to the participants who found knitting to be one of the absolute best ways for them to calm down during the day. Another participant used the calming benefits of knitting to help her in social situations. She remarked, “I'm a pretty introverted person so if I'm going to do something social I’ve feel like I need to be doing something” (participant 4). 
However, the soothing results of knitting got one participant into trouble:

first semester freshman year I knitted and crocheted more than I should have studied and so it kind of became a bad thing, "okay all I want to do is knit because I'm really stressed out.” But now for me it's kind of a reward like if I have a big test to study for I'll study for that and once I'm done taking the test I can come home and I'll let myself knit and then I can relax (participant 11).

Using knitting as a reward for a hard day's work was mention by a few other participants who enjoyed working on projects at the end of the day to unwind and allow themselves some creative time.

\section{Knitted Artifacts}

All but one participant brought multiple projects to share with the researcher during their interviews. To limit the sample size, participants were asked to choose their two favorite projects to be used in the material culture analysis. A total of twenty-nine items were selected for inclusion in the study. The items included in the material culture analysis were then compared to a similar set of items from www.ravelry.com to form conclusions.

\section{Materials}

The materials chosen by knitters to create their projects were analyzed in terms of color, fiber, and yarn weight; these were recorded into Table 4.3. Brown and green were tied for the most frequently utilized colors by participants with nine projects (18\%) each. The next most commonly used colors, with four projects (8\%) each, were black, orange, red, gold, and variegated. Surprisingly, the most popular color within the comparable sample was white/cream which had a total of eight items (18\%). Green was the second most often used color (13\%) by the comparable sample and the third choice was brown 
and grey which each had five projects (11\%). There was overlap between both samples as green and brown were highly utilized within each.

Twelve (35\%) of the participants chose to create their projects with wool yarn, which was the most popular pick. Acrylic was ranked second and had been used in six projects (17\%); cotton was used by four items (12\%) making it the third most prevalent choice. Wool was also widespread within the comparable sample which used it for eleven projects (34\%). Merino wool was significantly more popular within the comparable sample and was used in seven items (22\%).

With nine projects (31\%), over one third of the sample was comprised of worsted weight yarn. Bulky ranked as second (21\%) and DK was third (17\%). Not surprisingly, worsted was also the most popular yarn weight for the comparable sample; it was used for nearly half of the projects (48\%). However, fingering was chosen second most frequently (17\%) by the comparable sample. 
Table 4.3

Materials used in Knitted Artifacts

\begin{tabular}{|c|c|c|c|}
\hline \multicolumn{2}{|c|}{ Sample Knitted Artifacts $(n=29)$} & \multicolumn{2}{|c|}{ Comparable Knitted Artifacts ( $n=29)$} \\
\hline \multicolumn{2}{|c|}{ Colors Used in Items $(n=50)$} & \multicolumn{2}{|c|}{ Colors Used in Items $(n=45)$} \\
\hline Brown & $18 \%(n=9)$ & Brown & $11 \%(n=5)$ \\
\hline Green & $18 \%(n=9)$ & Green & $13 \%(n=6)$ \\
\hline Blue & $4 \%(n=2)$ & Blue & $9 \%(n=4)$ \\
\hline Black & $8 \%(n=4)$ & Black & $7 \%(n=3)$ \\
\hline Purple & $6 \%(n=3)$ & Purple & $4 \%(n=2)$ \\
\hline Orange & $8 \%(n=4)$ & Orange & $2 \%(n=1)$ \\
\hline Grey & $6 \%(n=3)$ & Grey & $11 \%(n=5)$ \\
\hline Red & $8 \%(n=4)$ & Red & $7 \%(n=3)$ \\
\hline White/Cream & $2 \%(n=1)$ & White/Cream & $18 \%(n=8)$ \\
\hline Gold & $8 \%(n=4)$ & Gold & $4 \%(n=2)$ \\
\hline Pink & $6 \%(n=3)$ & Pink & $9 \%(n=4)$ \\
\hline Variegated & $8 \%(n=4)$ & Variegated & $4 \%(n=2)$ \\
\hline \multicolumn{2}{|c|}{ Type of Yarn Used $(n=34)$} & \multicolumn{2}{|c|}{ Type of Yarn Used $(n=32)$} \\
\hline Acrylic & $17 \%(n=6)$ & Acrylic & $13 \%(n=4)$ \\
\hline Cotton & $12 \%(n=4)$ & Cotton & $6 \%(n=2)$ \\
\hline Silk & $0 \%(n=0)$ & Silk & $6 \%(n=2)$ \\
\hline Wool & $35 \%(n=12)$ & Wool & $34 \%(n=11)$ \\
\hline Mohair & $3 \%(n=1)$ & Mohair & $6 \%(n=2)$ \\
\hline Alpaca & $6 \%(n=2)$ & Alpaca & $9 \%(n=3)$ \\
\hline Merino Wool & $9 \%(n=3)$ & Merino Wool & $22 \%(n=7)$ \\
\hline Hemp & $3 \%(n=1)$ & Hemp & $0 \%(n=0)$ \\
\hline Wire & $3 \%(n=1)$ & Wire & $0 \%(n=0)$ \\
\hline Eco-friendly & $12 \%(n=4)$ & Eco-friendly & $\mathrm{n} / \mathrm{a}$ \\
\hline Cashmere & $0 \%(n=0)$ & Cashmere & $3 \%(n=1)$ \\
\hline \multicolumn{2}{|c|}{ Yarn Weight $(n=29)$} & \multicolumn{2}{|c|}{ Yarn Weight $(n=29)$} \\
\hline Lace & $0 \%(n=0)$ & Lace & $7 \%(n=2)$ \\
\hline Fingering & $7 \%(n=2)$ & Fingering & $17 \%(n=5)$ \\
\hline Sock & $3 \%(n=1)$ & Sock & $0 \%(n=0)$ \\
\hline Sport & $14 \%(n=4)$ & Sport & $3 \%(n=1)$ \\
\hline DK & $17 \%(n=5)$ & DK & $10 \%(n=3)$ \\
\hline Worsted & $31 \%(n=9)$ & Worsted & $48 \%(n=14)$ \\
\hline Bulky & $21 \%(n=6)$ & Bulky & $10 \%(n=3)$ \\
\hline Other & $7 \%(n=2)$ & Not Reported & $3 \%(n=1)$ \\
\hline
\end{tabular}




\section{Construction}

Different types of assembly methods were analyzed within the construction portion of the material culture analysis, such as the method of constructing a project and favored techniques; these are displayed in Table 4.4. Within the participant artifact sample flat knit was most prevalent, nineteen (66\%) of the projects had been constructed in that manner. The remaining ten items (34\%) had been knit in the round either on double pointed needles or circular needles. The split between flat knit (48\%) and knitting in the round (52\%) was more even in the comparable data set.

Stockinette was by far the favorite stitch in the sample artifacts, it was used for fifteen items (28\%) and comprised almost a third of all the techniques employed. The next most widely used construction was rib stitch (13\%), possibly because it is a method of creating a very stretch fabric that it often used at hems or other areas to fit closely. Other top stitches were lace (11\%) and cables (9\%). A similar ranking of favorite techniques was seen in the comparable data set. Again, stockinette was the favored stitch with twenty items (33\%) and rib stitch was ranked second with ten (17\%). Lace was still ranked as the third most often utilized technique with eight total projects (13\%). However, garter stitch was more important within the comparable data set and was tied with lace for the third most often used stitch (13\%). 
Table 4.4

Constructions used in Knitted Artifacts

\begin{tabular}{|c|c|c|c|}
\hline \multicolumn{2}{|c|}{ Sample Knitted Artifacts $(n=29)$} & \multicolumn{2}{|c|}{ Comparable Knitted Artifacts $(n=29)$} \\
\hline \multicolumn{2}{|c|}{ Type of Construction Used $(n=29)$} & \multicolumn{2}{|c|}{ Type of Construction Used $(n=29)$} \\
\hline Flat Knit & $66 \%(n=19)$ & Flat Knit & $48 \%(n=14)$ \\
\hline Knit in the Round & $34 \%(n=10)$ & Knit in the Round & $52 \%(n=15)$ \\
\hline \multicolumn{2}{|c|}{ Type of Technique Used $(n=55)$} & \multicolumn{2}{|c|}{ Type of Technique Used $(n=60)$} \\
\hline Stockinette & $28 \%(n=15)$ & Stockinette & $33 \%(n=20)$ \\
\hline Rib Stitch & $13 \%(n=7)$ & Rib Stitch & $17 \%(n=10)$ \\
\hline Cables & $9 \%(n=5)$ & Cables & $7 \%(n=4)$ \\
\hline Lace & $11 \%(n=6)$ & Lace & $13 \%(n=8)$ \\
\hline Garter & $7 \%(n=4)$ & Garter & $7 \%(n=4)$ \\
\hline Color Work & $4 \%(n=2)$ & Color Work & $3 \%(n=2)$ \\
\hline Basket Stitch & $5 \%(n=3)$ & Basket Stitch & $2 \%(n=1)$ \\
\hline Seed Stitch & $5 \%(n=3)$ & Seed Stitch & $0 \%(n=0)$ \\
\hline Braids & $4 \%(n=2)$ & Braids & $2 \%(n=1)$ \\
\hline Stripes & $5 \%(n=3)$ & Stripes & $7 \%(n=4)$ \\
\hline Felting & $2 \%(n=1)$ & Felting & $0 \%(n=0)$ \\
\hline Closures & $7 \%(n=4)$ & Closures & $10 \%(n=6)$ \\
\hline
\end{tabular}

\section{Provenance}

The provenance section examined the date when the artifact was constructed; the summary is in Table 4.5. The majority of the participants had made their knitted items in either January-February of 2011 (31\%) or June-December of 2010 (31\%). Another four (14\%) artifacts were created between January-June 2010. It was the opposite within the comparable data section. The majority of the items were created before 2009 (59\%). Unfortunately, another six items (21\%) did not record any sort of date of completion which could have greatly impacted the timeline. 
Table 4.5

Provenances of Knitted Artifacts

\begin{tabular}{|lr|lr|}
\hline \multicolumn{2}{|l|}{ Sample Knitted Artifacts $(n=29)$} & \multicolumn{2}{l|}{ Comparable Knitted Artifacts $(n=29)$} \\
\hline \multicolumn{2}{|l|}{ When was Item Produced $(n=29)$} & \multicolumn{2}{l|}{ When was Item Produced $(n=29)$} \\
Jan '08-June '08 & $3 \%(n=1)$ & Before 2008 & $28 \%(n=8)$ \\
July '08-Dec '08 & $7 \%(n=2)$ & Jan '08-June '08 & $10 \%(n=3)$ \\
Jan '09-June '09 & $7 \%(n=2)$ & July '08-Dec '08 & $21 \%(n=6)$ \\
July '09-Dec '09 & $7 \%(n=2)$ & Jan '09-June '09 & $0 \%(n=0)$ \\
Jan '10-June '10 & $14 \%(n=4)$ & July '09-Dec '09 & $7 \%(n=2)$ \\
July '10-Dec '10 & $31 \%(n=9)$ & Jan '10-June '10 & $3 \%(n=1)$ \\
Jan '11-Feb '11 & $31 \%(n=9)$ & July '10-Dec '10 & $3 \%(n=1)$ \\
& & Jan '11-Feb '11 & $7 \%(n=2)$ \\
& & Not Reported & $21 \%(n=6)$ \\
\end{tabular}

Provenances also looked that the various reasons for the item to have been made, which are displayed in Table 4.6. A few participants gave multiple reasons for the creation of the item. For example, one woman had made a hat to learn how and also gave it as a gift. The items in the participant sample were predominantly used for gifts; nine items (23\%) were meant for this purpose. Next, every day wear (20\%) and winter wear (18\%) were highly ranked. A total of five items (13\%) were constructed so that participants could learn new stitches or techniques. As the participant was present while the knitted artifacts were studied the researcher was able to ask more specific questions about the provenance of the item which was not available for the comparable data set. The majority (56\%) of the comparable artifacts appeared to be for everyday wear. Winter wear was also popular and eight items (26\%) seemed to be for cold weather. Contrary to the sample artifacts, four (13\%) of the comparable items seemed to be for special occasion use such as to compliment evening wear. 
Table 4.6

Reasons for Making Knitted Item

\begin{tabular}{|c|c|c|c|}
\hline \multicolumn{2}{|c|}{ Sample Knitted Artifacts $(n=29)$} & \multicolumn{2}{|c|}{ Comparable Knitted Artifacts $(n=29)$} \\
\hline \multicolumn{2}{|c|}{ Reason for Making Item $(n=39)$} & \multicolumn{2}{|c|}{ Reason for Making Item $(n=31)$} \\
\hline Every Day Wear & $20 \%(n=8)$ & Every Day Wear & $52 \%(n=16)$ \\
\hline Winter Wear & $18 \%(n=7)$ & Winter Wear & $26 \%(n=8)$ \\
\hline Gifts & $23 \%(n=9)$ & Gifts & $\mathrm{n} / \mathrm{a}$ \\
\hline Match a Garment & $7 \%(n=3)$ & Match a Garment & $\mathrm{n} / \mathrm{a}$ \\
\hline Fall Wear & $5 \%(n=2)$ & Fall Wear & $\mathrm{n} / \mathrm{a}$ \\
\hline Summer/Spring Wear & $5 \%(n=2)$ & Summer/Spring Wear & $\mathrm{n} / \mathrm{a}$ \\
\hline Humor & $3 \%(n=1)$ & Humor & $3 \%(n=1)$ \\
\hline Decoration & $3 \%(n=1)$ & Decoration & $6 \%(n=2)$ \\
\hline Special Occasion & $3 \%(n=1)$ & Special Occasion & $13 \%(n=4)$ \\
\hline Learn Stitches & $13 \%(n=5)$ & Learn Stitches & $\mathrm{n} / \mathrm{a}$ \\
\hline
\end{tabular}

\section{Function}

The category dedicated to function explored the type of items created, the efficacy of those items, and also the intended users; these are available in Table 4.7, Table 4.8, and Table 4.9. At this point it would be good to remember that items in the comparable data section were chosen to mimic the sample artifacts; therefore there is no difference between the types of items in each sample. Hats and scarves were the most common project, each were made six times (21\%) and accounted for over forty percent of the entire sample. The other most popular items were gloves/mittens and sweaters/cardigans, both were made three times (11\%) each. 
Table 4.7

Function of Knitted Artifacts

\begin{tabular}{|lr|lr|}
\hline \multicolumn{2}{|l|}{ Sample Knitted Artifacts $(n=29)$} & Comparable Knitted Artifacts $(n=29)$ \\
& & & \\
Type of Item $(n=29)$ & & Type of Item $(n=29)$ \\
Sweater/Cardigan & $11 \%(n=3)$ & Bag & $7 \%(n=2)$ \\
Socks & $3 \%(n=1)$ & Sweater/Cardigan & $11 \%(n=3)$ \\
Headband/Scarf & $7 \%(n=2)$ & Socks & $3 \%(n=1)$ \\
Scarf & $21 \%(n=6)$ & Scadband/Scarf & $7 \%(n=2)$ \\
Belt & $3 \%(n=1)$ & Belt & $21 \%(n=6)$ \\
Hat & $21 \%(n=6)$ & Hat & $3 \%(n=1)$ \\
Cowl & $7 \%(n=2)$ & Cowl & $21 \%(n=6)$ \\
Toy & $3 \%(n=1)$ & Toy & $7 \%(n=2)$ \\
Leg Warmers & $3 \%(n=1)$ & Leg Warmers & $3 \%(n=1)$ \\
Home Decor & $3 \%(n=1)$ & Home Decor & $3 \%(n=1)$ \\
Gloves/Mittens & $11 \%(n=3)$ & Gloves/Mittens & $3 \%(n=1)$ \\
& & & $11 \%(n=3)$ \\
\hline
\end{tabular}

The researcher surveyed the success of the final items; again the participants occasionally used more than one word to describe their final project. Ten items (31\%) were identified as having great fit. Another quarter of the projects were found to be cute (12\%) or the participants claimed that they loved it (15\%). However, not all the items were considered to be a success; twelve items (37\%) did not fulfill the exact purpose that the participants had intended, ten items had some sort of fit mistake (31\%). Fit mistakes included items that were the wrong size; it also encompassed items that had been constructed incorrectly. For example, one woman had made a sweater using a certain type of neckline, but after it was completed she decided that the neckline looked silly and she decided to take it apart and replace it with a different style. It was not possible to 
determine the effectiveness of the comparable artifacts, most of which had only a single picture to judge by.

Table 4.8

Efficacy of Knitted Artifacts

\begin{tabular}{|c|c|c|c|}
\hline \multicolumn{2}{|c|}{ Sample Knitted Artifacts $(n=29)$} & \multicolumn{2}{|c|}{ Comparable Knitted Artifacts $(n=29)$} \\
\hline Was Item Effecti & & Was Item Effect & \\
\hline Good Fit & $31 \%(n=10)$ & Good Fit & $\mathrm{n} / \mathrm{a}$ \\
\hline Cute & $12 \%(n=4)$ & Cute & $\mathrm{n} / \mathrm{a}$ \\
\hline Warm & $6 \%(n=2)$ & Warm & $\mathrm{n} / \mathrm{a}$ \\
\hline Loves It & $15 \%(n=5)$ & Loves It & $\mathrm{n} / \mathrm{a}$ \\
\hline Itchy & $3 \%(n=1)$ & Itchy & $\mathrm{n} / \mathrm{a}$ \\
\hline Fit Mistake & $31 \%(n=10)$ & Fit Mistake & $\mathrm{n} / \mathrm{a}$ \\
\hline Needs Blocking & $3 \%(n=1)$ & Needs Blocking & $\mathrm{n} / \mathrm{a}$ \\
\hline
\end{tabular}

The final area in function surveyed the intended users of the knitted artifacts.

Within the sample data there were eighteen (62\%) artifacts intended to be used the knitter. Three (11\%) were meant for class projects. The remaining eight items were gifts and were meant to be used by a number of different people, husbands/boyfriends, mothers, nieces, friends, and swap partners. Originally, in the provenances section there were nine gifts but one knitter decided to keep a project that started out as a gift, dropping the total number of gifts to eight. The specific end users of the artifacts in the comparable data set were less clear. Twenty two of the items were meant for women (75\%) and four items (14\%) were for men. 
Table 4.9

Intended User of Knitted Artifacts

\begin{tabular}{|lr|lr|}
\hline \multicolumn{2}{|l|}{ Sample Knitted Artifacts $(n=29)$} & \multicolumn{2}{l|}{ Comparable Knitted Artifacts $(n=29)$} \\
\hline Intended User $(n=29)$ & & Intended User $(n=29)$ & \\
Self & $62 \%(n=18)$ & Women & $75 \%(n=22)$ \\
Husband/Boyfriend & $7 \%(n=2)$ & Men & $14 \%(n=4)$ \\
Niece & $7 \%(n=2)$ & Children & $11 \%(n=3)$ \\
Friend & $3 \%(n=1)$ & & \\
Mom & $7 \%(n=2)$ & & \\
Swap Partner & $3 \%(n=1)$ & & \\
Class Project & $11 \%(n=3)$ & & \\
& & & \\
\hline
\end{tabular}

\section{Value}

The participant sample had quite a range of values associated with their products including monetary, emotional, and societal; the values are located in Table 4.10. The typical amount spent on projects was \$10-19 (24\%). Fifteen (51\%) of the projects cost under $\$ 9$ to knit, this may have been because of the relatively lower incomes made by the majority of the participants. It was not possible to locate the final total cost of materials for the comparable items, but as the projects had come from the patterns section of www.ravelry.com it was possible to determine how much the pattern itself cost. Nineteen (66\%) of the patterns used to make the comparable artifacts were available for free. Another nine (32\%) were available for less than $\$ 9$.

For emotional value there were nine artifacts (31\%) which were intended to become gifts within the sample artifacts. Three knitted artifacts (11\%) were created as part of class room projects. "Firsts" such as the first project or first sweater completed were also highlighted by participants. Determining the emotional value of the 
comparable artifacts was difficult, without speaking to the knitters it was not possible to determine if they were gifts or "firsts." However, as the artifacts in the comparable data section came from the patterns section all of the items (100\%) had been used to inspire other knitters to create the same artifact.

In terms of social value there were two items (7\%) inspired by popular culture and two items (7\%) made from recycled materials within the sample artifacts. A total of three items (11\%) in the comparable data set were also inspired by popular culture. However, it was not possible to determine if any of the items had been constructed from recycled materials. 
Table 4.10

Value of Knitted Artifacts

\begin{tabular}{|c|c|c|c|}
\hline \multicolumn{2}{|c|}{ Sample Knitted Artifacts $(n=29)$} & \multicolumn{2}{|c|}{ Comparable Knitted Artifacts ( $n=29)$} \\
\hline \multicolumn{2}{|c|}{ Materials Cost of Item $(n=29)$} & \multicolumn{2}{|c|}{ Pattern Cost of Item $(n=29)$} \\
\hline Free & $17 \%(n=5)$ & Free & $66 \%(n=19)$ \\
\hline$<\$ 5$ & $17 \%(n=5)$ & $<\$ 5$ & $11 \%(n=3)$ \\
\hline$\$ 5-\$ 9$ & $17 \%(n=5)$ & $\$ 5-\$ 9$ & $21 \%(n=6)$ \\
\hline$\$ 10-\$ 19$ & $24 \%(n=7)$ & $\$ 10-\$ 19$ & $3 \%(n=1)$ \\
\hline$\$ 20-\$ 29$ & $14 \%(n=4)$ & $\$ 20-\$ 29$ & $0 \%(n=0)$ \\
\hline$\$ 30-\$ 39$ & $3 \%(n=1)$ & $\$ 30-\$ 39$ & $0 \%(n=0)$ \\
\hline$\$ 40-\$ 49$ & $3 \%(n=1)$ & $\$ 40-\$ 49$ & $0 \%(n=0)$ \\
\hline$>\$ 50$ & $3 \%(n=1)$ & $>\$ 50$ & $0 \%(n=0)$ \\
\hline \multicolumn{2}{|c|}{ Emotional Value of Item $(n=29)$} & \multicolumn{2}{|c|}{ Emotional Value of Item $(n=29)$} \\
\hline Gift & $31 \%(n=9)$ & Gift & \\
\hline Inspired Others & $3 \%(n=1)$ & Inspired Others & $100 \%(n=29)$ \\
\hline Class Work & $11 \%(n=3)$ & Class Work & $\mathrm{n} / \mathrm{a}$ \\
\hline Firsts & $7 \%(n=2)$ & Firsts & $\mathrm{n} / \mathrm{a}$ \\
\hline \multicolumn{2}{|c|}{ Societal Value of Item $(n=29)$} & \multicolumn{2}{|c|}{ Societal Value of Item $(n=29)$} \\
\hline Popular Culture & $7 \%(n=2)$ & Popular Culture & $11 \%(n=3)$ \\
\hline Recycled & $7 \%(n=2)$ & Recycled & \\
\hline
\end{tabular}




\section{CHAPTER FIVE DISCUSSION}

\section{Introduction}

The research was conducted in Northern Colorado and examined the motivations for participation and meaning of knitting in the lives of young women (ages 18-30). The researcher employed Uses and Gratifications (UG) theory and five categories determined by Katz et al. (1974), cognitive, affective, personal integrative, social integrative, and tension release, to better understand the participants range of incentives for engaging in knitting. Applying UG to the knitters within the sample allowed the researcher to describe the specific culture of the participants in terms of motivations and benefits. A material culture analysis defined by Smith (1985) was used to further examine the motives, decisions, and cultural meanings of the knitted artifacts produced by the women. Demographic and knitting preference data were also collected via the Post Interview Participant Survey (PIPS) to cross check what was discovered in the interviews.

\section{Interview Data}

Data collected through the interviews were analyzed using the three stages of coding that are part of constant comparative method. Three major themes emerged from the data and thirteen sub-themes were also identified. 
The first major theme was incentives to participate in knitting. The theme included allowing for creativity and the ability to multi-task in the daily lives of knitters. Second, financial incentives, such as free or inexpensive yarn also contributed to participation in the knitting process. The participants were excited to create unique items through conducting alterations and using various information sources, such as books and websites were used to obtain patterns and knitting knowledge. The final motivator for the knitters was the diverse social aspects associated with the craft, for instance knitting circles and knitting events.

The second major theme was barriers which represented the obstacles participants had to overcome to partake in their hobby. Many of the participants had encountered some sort of negative reactions from both strangers and friends or family members. Mistakes caused a number of projects to be abandoned and sometimes caused the knitters to doubt themselves and their abilities. The expense of materials also served as a barrier to knitting.

The third major theme was outcomes, which was comprised of the results that encouraged continued involvement. Positive reactions from others such as compliments helped to build the confidence of the knitters. The resulting products were highly regarded and fostered a sense of pride. Relaxation was the most regularly mentioned and sought after results of the knitting process.

Connections between overarching themes and amid subthemes can be made. For example, a link between the major themes of outcomes and incentives was observed. Positive outcomes regularly served as motivators for the participants to continue knitting. For instance, “I find that if I know that I’m going to get a reaction from somebody on a 
knitted project I get like ten times more excited to sit down and work on it, finish it as fast as I can” (participant 11). In the quote the participant anticipated a positive outcome from her project which in turn motivated her to finish knitting the project. Another example was relaxation that resulted from the physical process of knitting often caused the women to continue knitting in order to maintain the resultant stress relief. Similarly, some incentives to knitting could also be seen as benefits. Making friends through knitting circles was seen as not only a motivator, but also as a constructive outcome for the participants.

An association can be made between the social aspects of knitting and the positive reactions knitters enjoyed. Participation in knitting groups and in online communities led to compliments on knitting projects. Many of the women enjoyed other knitters because they were so encouraging and supportive of their work. The backing of other knitters often led to increased confidence in the participants which in turn drove them to participate more and to try increasingly difficult stitches and patterns.

The expense related to knitting supplies was mentioned as a major concern and an obstacle to knitting. Due to the cost of yarn many of the knitters had developed alternative purchasing habits to reduce the financial burden of knitting. They shopped at thrift stores, used gifts, and bought extra yarn at sales. Yarn not purchased for an immediate project was placed into their crafting stash for future projects. By purchasing materials at a discount and storing them for future work the knitters were able to get better prices for yarns which allowed for further involvement. 


\section{Knitted Artifacts}

The material culture data were analyzed using the methods defined by Smith (1985). This portion of the study yielded some interesting results, as a whole knitting smaller, portable projects was favored by the participants. Scarves and hats were most often brought for inclusion in the study. The overall preference for portable accessory projects may have related to the involvement of the participants in public knitting and events like "Knit Night.” During these events it was necessary to bring an entire project; however, limited space in some places could have dictated the size of a project to be comfortably worked in that space. The knitters socialized or listened to others which divided their attention. The level of involvement in multiple tasks while knitting also had an effect on the projects the participants felt comfortable with trying to work. For example, “for the most part if it’s a really complicated pattern then I don't take it out in public because I need to focus on that” (participant 4).

The favored fiber was wool. A few of the knitters explained their preference for natural fibers over synthetics. For example, “I do usually chose wool I don’t like working with synthetic, every time I see something that's a certain percentage of synthetic I don't like to get it” (participant 12). Wool is readily available at many yarn retailers, it comes in a number of colors, and it can also be certified organic which was of importance to the participants (Minahan \& Wolfram Cox, 2007; Zawilinski, 2010).

There was also a preference for making artifacts using the colors brown and green. It is possible that these color choices reflect further attention and interest in sustainability which is often associated with natural colors. The most used yarn weight was worsted. Worsted weight yarn is the most readily available type of yarn on the 
market and a large number of knitting patterns are gaged for that type of yarn, which probably accounts for it being the most frequently utilized yarn weight (Stoller, 2003).

The top three stitches used included stockinette, cables, and lace. The popularity of stockinette was expected; it is one of the most versatile of all stitches and is used in numerous patterns for small sections such as the heels or socks or for entire garments like sweaters (Colton, 1979). Cables are another popular type of stitch that is relatively easy to work but creates a complex texture which is visually interesting (Hollingsworth, 1982). The other favorite was lace stitch which has been termed as "the height of the knitters' art," the inclusion of lace elevates the difficulty of a pattern providing a challenge for the knitters (Walker, 1971, p. 5). The participants often spoke of enjoying challenges in their knitting as it was fun to figure out how to complete the pattern and fostered a sense of accomplishment upon completion.

July 2010- February 2011 was the most popular time frame for artifacts to be completed. The recent completion of many of the artifacts related to the inclination of the participants towards giving their projects as gifts. A number of the participants remarked that they hardly kept anything for themselves; so many of the artifacts were recently completed and on hand because they had yet to be given away.

Nearly half of the items were created for either gifts and/or everyday wear. In terms of efficacy of the final product, one third of the items were identified as having great fit and one third had fit mistakes. The majority of the participants were less concerned with mistakes in their projects, which has been described as a characteristic of knitters by researchers (Rosner \& Ryokai, 2009). 
A majority of items were made for the knitter themselves and half of the projects had cost less than nine dollars to create. The reasonable cost of materials for the artifacts often resulted from the knitters' use of stashed yarns. Using stash materials to minimize costs associated with making handmade items has been described by other researchers (Pentney, 2008; Turney, 2004). In addition, the participants were creating more accessories so there was less need for extensive yardage like larger projects which helped to minimize the overall cost of artifacts.

\section{Participant Demographics}

Data collected from the PIPS were analyzed by running frequencies and descriptive statistics using Statistical Package for the Social Sciences (SPSS). Only fourteen of the knitters participated in the PIPS, they ranged in age from 20 to 29 years old. All of the women had at least some college experience and half had obtained a bachelor's degree. The age and education level of the sample participants is indicative of what has been described as "new” knitters who are young, well educated women (Myzelev, 2009; Stoller, 2003). The majority of the sample made under $\$ 25,000$ per year. These young, female knitters have been identified as having relatively low income in other research (Chansky, 2010). Nearly half had been knitting for less than three years and the others had been knitting for over five years. Specific years of experience have not been described by other researchers, however, some have described that a number of young, female knitters may have learned fairly recently from friends and knitting group members (Minahan \&Wolfram Cox, 2007; Parkins, 2004).

Previous studies have not described the specific knitting involvement and skill levels of young, female knitters so it was not possible to determine how the sample 
related to other knitters on these factors. The knitters spent anywhere between nine hours to less than one hour a week working on their projects. In terms of skill level, half of the women rated themselves as intermediates.

The sample was fairly evenly split between process and product knitters. The process of knitting has been cited as one of the most enjoyable parts of knitting (Myzelev, 2009). The resulting products from the knitting process have also been identified as an extremely important outcome (Schofield-Tomschin \& Littrell, 2001). Both the process and products that come from knitting have been identified as important, however no research has specifically found that certain knitters identify as one or the other.

The majority of the participants spent under \$25 a month on knitting supplies. Again, using stashed materials and seeking out sales was one method for participants to reduce the overall costs associated with knitting (Pentney, 2008; Turney, 2004). All of the knitters had purchased wool yarn in the past year and over three quarters had also purchased acrylic yarn. Again, wool is readily available, easy to obtain, and can be organic (Zawilinski, 2010). In terms of preferred techniques, knitting in the round was preferred over flat knit and cables were also rated highly. No other research has discovered the preferred construction techniques for knitters to utilize.

Scarves were ranked as the most frequently made project by the participants in the past year followed by hats, and gifts. Scarves and hats are some of the most common projects for young, female knitters; in addition, these projects were also suitable for beginner or intermediate knitters which correlated with the skill level of the majority of the participant sample (Hiatt, 1988; Minihan \& Wolfram Cox, 2007). Gifts are also very 
popular for handcrafters to create as they forge bonds with recipients and demonstrate that the recipient is highly valued by the crafter (Johnson \& Wilson, 2005; Turney, 2004).

\section{Research Questions}

Four research questions were identified to be addressed during the research

process. All of the questions were attended to throughout the data collection process.

For this study the subsequent research questions were identified:

1. What are the motivations, benefits, and meanings of knitting for young women? How do these relate to Uses and Gratifications Theory?

2. What types of products are young, female knitters constructing?

3. How do the physical artifacts that are produced by young, female knitters relate to their motivations for engaging in knitting?

4. How do the physical artifacts that are produced by young, female knitters relate to their specific social and political culture?

\section{Research Question Number One}

What are the motivations, benefits, and meanings of knitting for young women? How do these relate to Uses and Gratifications Theory? Research question one was answered through the data gathered from the in depth interviews with participants, the material culture analysis, and the PIPS. The theme of incentives related closely to motivations for engaging in knitting. In turn, the theme of positive outcomes examined some of the potential benefits.

\section{Motivations}

Knitters cited a variety of reasons for engaging in the craft. Knitting presented an opportunity for the participants to engage in a creative process. Creating personalized items allows knitters to feel special and unique (Pentney, 2008). The creative potential of 
knitting was highlighted as important in today's society because globalization has caused many products to become homogenized (Morgado, 1996). Some of the knitters commented that making their own garments and accessories allowed them to follow their own creative vision as opposed to the vision of retailers. They observed that items similar to what they had produced were not available for purchase. The pride knitters felt in differences between their hand knits and mass produced items has also been observed by other researchers (Rosner \& Ryoki, 2009).

Social events and knitting circles helped to motivate and provided a support system for knitters. The social aspects of knitting were highlighted by researchers as enormously important for receiving support for crafting and for enjoying camaraderie (Parkins, 2004; Schofield-Tomschin \& Littrell, 2001). A number of these events and circles were on the Internet which allowed a greater number of knitters to be involved. Online events and groups foster a sense of group identity among knitters by using a shared language and repeated interactions which strengthen the bonds between the knitters as well (Pentney, 2008).

Knitting circles were also identified as one of the places where techniques were passed down to new generations which may not have learned their skills from family members (Hawley, 2005; Minahan \& Wolfram Cox, 2007). The participants were able to learn new skills at knitting groups, but unlike in the literature, many of the knitters had learned skills from immediate female family members such as mothers, mother-in-laws, sisters, and aunts. Surprisingly, books and the Internet were also utilized by a few participants to teach themselves how to knit. 


\section{Benefits}

The potential benefits from knitting were also emphasized by participants during their interviews. The major benefit for many knitters was the actual products they created through their crafting. Positive reactions from other knitters and outsiders further enhanced their enjoyment of the knitting process. Both the products and positive reactions led to increased confidence within the knitters in their abilities and skill set. Researchers have also documented the positive results that resulted from compliments to final projects (Johnson \& Hawley, 2004; Schofield-Tomschin \& Littrell, 2001).

Relaxation was another key benefit of the knitting process. All of the women described knitting as a great stress reliever and some continued to knit in order to prolong the relaxation that resulted from the activity. A number of the participants utilized knitting to entertain themselves which allowed them to focus on other tasks such as conversations, lectures, and movies. The stress relief and alternative meditative benefits that could be gained from the knitting process has also been highlighted by researchers (e.g. MacDonald, 1988; Parkins, 2004).

\section{Meanings}

The participants tended to identify themselves as one of two types of knitters either process of product knitters. Benefits from both the process and final products were described by researchers; however, no other research has described the idea of process and product knitters (Myzelev, 2009; Schofield-Tomschin \& Littrell, 2001). The process knitters described themselves as chiefly interested in the physical act of creating items and less with the final outcome. For instance one process knitter explained, “mostly I make things and give them away, I like the process” (participant 8). Clearly the knitter 
had less interest in the final outcomes as she was giving the majority of her items away after finishing the projects.

Whereas the product knitters were concerned with the final result from their efforts and had less enjoyment from the actual knitting process. One product knitter stated, "I guess for me I'm more motivated by the finished product than the process, I know some people are the exact opposite, but I want it to be beautiful and perfect and done” (participant 3). Whether a participant identified as a product or process knitter had a great impact on the actual meaning on knitting in their lives.

It was stated that knitting while watching television and doing other activities fostered a sense of productivity. Most of the participants felt they needed to keep their hands busy in order to improve focus and to allow them to fully connect with whatever task they were engaging in. This was in opposition to the claim that knitting was representative of a yearning for a slower more balanced life as the need to feel productive at all times is indicative of fast paced postmodern lifestyles (Parkins, 2004).

On a related note, other knitters felt that they were able to be self-sustained though their efforts. They were pleased to discovered it was possible for them to "make my own” as oppose to being forced to consume. One knitter discussed how she enjoyed knitting her own things as it linked her to the history of knitting and homemade. She stated, “this is what people used to do, you had to do it because machines didn't make it before there were machines" (participant 1). The feeling that handmade was superior to mass produced items has been noticed by researchers as well, who found some contemporary knitters were concerned with helping others to appreciate hand knitted items (Rosner \& Ryokai, 2009). 
The wide availability and use of the internet to forward knitting was one of the most important aspects of the craft to the participants. The knitters in the sample were incredibly aware of and dependent on the Internet to further their crafting. They used online videos to learn new stitches, shared patterns with one another over social networking sites, and posted messages to other knitters worldwide which created supportive social climate for knitting. Websites like www.ravelry.com were crucial to many of the knitters who believed it would be impossible for them to continue knitting without the virtual knitting community. The increasing availability of information sources such as the Internet and retail establishments was referred to in the literature base. The use of the Internet to join knitting circles, get help, and find patterns was suggested as an important support for knitters to gain knowledge and continue to participate in the craft (Minahan \& Wolfram Cox, 2007).

\section{Application to Theory}

The motivations and benefits of knitting were interconnected and often worked in a circular process wherein motivations led to benefits which further encouraged knitting. The incentives and outcomes enjoyed by the participants were closely aligned with UG and the categories as suggest by Katz et al. (1974). Knitting was able to fulfill all five of the categories for the women.

The participants were able to fulfill cognitive needs by knitting. There were women who were chiefly concerned with understanding how knitting worked and how stitches would fit together to make patterns. For example, "I think it’s challenging, but I like being able to figure it out; it’s sort of like a puzzle” (participant 12). The puzzle of knitting patterns and stitches were endlessly fascinating for some of the knitters. 
Oftentimes changing patterns required knitters to use graph paper to map changes; this also enabled them to better understand the intricacies of patterns and how stitches fit together within projects. Numerical thinking such as mathematics and counting to help understand how to make knitted items was used by participants to change patterns is associated with cognitive thought (Edwards, 1999).

Participants were able to use their knitting to fulfill affective needs by allowing them to be creative in their daily lives. Generating a creative, unique product was accompanied by an intense level of pleasure and motivation to continue working on their project which Csikszentmihalyi (1990) described as flow. As is typical with activities that once produced a flow state, the knitters quickly became bored and had to seek out new skills to prolong their enjoyment of the activity. One knitter commented, "now I'm at this point where I'm sick of scarves and I just want to try, there's so many projects that I want to do" (participant 7). Other research has noted the same need for crafters to increase their skills in order to prolong flow and the enjoyment of their chosen activity (Blood, 2006).

Knitting was also used to satisfy personal integrative needs. Originally, the researcher postulated that knitters would satisfy these needs by gaining status within the knitting community via blogs and winning contests (Pentney, 2008). However, it soon became clear that competition and status among the knitters was not particularly encouraged. Several participants enjoyed being part of knitting circles as there was a lack of competitive spirit within the group. However, personal integrative needs were still met, but by the larger public as opposed to the knitting community. Members of the public often offered compliments; some were even shocked and amazed that the 
participants were able to knit. The compliments from members of the community created an opportunity for the knitters to say, “I made it!” The women shared that able to say they had made their items was one of their favorite parts of the knitting process. Making handcrafted items helps to "instill a sense of pride, value, and status in the maker" (Turney, 2004, p. 278).

Social integrative needs were met by women who participated in knitting circles as well as the online knitting community. One knitter commented, "I do enjoy knitting with other people because it's nice to just be able to talk and knit with people that actually understand what you're doing” (participant 10). Several participants identified involvement with other knitters as the best part of knitting. The enjoyment knitters felt from sharing understanding, excitement, and their projects with one another has been described by many researchers (Minihan \& Wolfram Cox, 2007; Schofield-Tomschin \&Littrell, 2001). However not all of the women were socially motivated, some of the participants self-identified as solitary knitters and were happier practicing their hobby alone. Solitary knitters have not been specifically described by any other researchers. Tension release or entertainment needs were fulfilled by knitting as well. The relaxation and stress relief that resulted from the knitting process was deemed as vital by all the participants. The stress relief that occurs from knitting results from physiological changes such as a reduced heart rate and also from psychological changes such as taking time for the self (Hollows, 2003; Reynolds, 1997; Reynolds, 2009). The ability to knit simultaneously with other tasks allowed them to stay entertained and also to feel productive. For instance: 
It's nice to be able to watch a movie or TV and then knit at the same time, it's always been nice and then I don't feel like I just wasted a ton of time, I have to be productive (participant 11).

Excitement over the ability to multi-task while knitting has been described by researchers as an attractive feature of knitting (Myzelev, 2009; Parkins, 2004).

\section{Research Question Number Two}

What types of products are young, female knitters constructing? Question two was answered primarily by the material culture analysis, but the interviews and PIPS also provided information. Due to the limited literature base and the lack of studies focused on the material culture of knitters, it was not possible to compare the items the participants were knitting to other research. The knitters in this sample felt the items they created were fashionable. It was stated that their projects were in opposition to what the general public envisioned as hand knitted items. One woman reflected, “I don’t think that they think about like you can have a headband or a cute shirt, they just think like scarves and knitted rugs” (participant 6). Interestingly, one participant described a visible difference in products made by young knitters in opposition to older knitters. She claimed, “a lot of the younger knitters I know do a lot more like novelty items like the little toys or the cute little Christmas ornament thing” (participant 2).

There was a general preference for creating accessories such as: toys, headwear, scarves, mittens, and bags, among the participants. According to the PIPS, more than half (57\%) of the total items made by the participants in the past year could be classified as accessories. These items were preferred as they were portable which was extremely important for knitters who enjoyed public knitting. In fact, one knitter was making a pair of fingerless gloves and carried all her supplies in a child's lunchbox. She stated, "this is 
a really great project because it's portable I’ve been working on it everywhere, library, student center, at home, on the bus, coffee places” (participant 7). While it would be easy to assume that product knitters would be more interested in quick accessories projects, there was not a specific preference of items made by process or product knitters.

Garments were less popular, but the participants still produced items like sweaters, dresses, and vests. Only a quarter (24\%) of the total items made by the participants over the past year could be counted as garments. These items were perceived as being more difficult to create and some of the participants were hesitant to start a garment project because they felt unsure of their skills in terms of knitting larger items. Garments were also avoided because of the large amount of yarn necessary to build them and thus the increased cost of the project. Finally, garments may have been started by participants, but as soon as the project grew too big to be portable they worked on it significantly less, which made it take longer to finish.

A number of the knitted artifacts were slated to become gifts. Gifts were quite common among the knitters, nine (23\%) of the items in the sample were slated to be presents. When an individual gives a handmade gift it is meant as more than just the item, it is the time, effort, and thoughts of the maker as well (Johnson \& Wilson, 2005). According to the PIPS, nine (64\%) of the knitters had made gifts in the past year. Oftentimes the knitters had to fight their impulses to give in order to keep some of their artifacts. Giving gifts, “that's my downfall, I make lovely things and then give them away, I'm trying to hold on to these even though I know that they'd fit my mom much better than me” (participant 7). Others coveted the items that they had originally started 
as gifts and decided to keep them. One woman admitted, "sometimes you get so attached that you're like, do I really like this person enough to give them this?” (participant 15).

\section{Research Question Number Three}

How do the physical artifacts that are produced by young, female knitters relate to their motivations for engaging in knitting? Research question three was addressed by the data gathered in the material culture analysis and interviews. The artifacts produced by the knitters related to their motivations for participating in knitting in a number of ways. Some knitters were more focused in terms of motivation whereas others were a composite of multiple categories.

Cognitive motivations affected the products made by the knitters in a couple of ways. Some of the knitters were interested in making groups of projects off the same pattern, for example, “I’ve made several of these hats and I'll change the (stitch) pattern on it just to see if I can get the pattern to work" (participant 12). With each new item, the knitter would try a new stitch pattern and adjust the number of total stitches to accommodate. A couple knitters explained that it was very difficult for them to envision the result of a pattern so they would often knit a test garment, evaluate and then adjust the math according to the desired fit. No other researchers have described creating test garments or interchanging different stitches into patterns and how they relate to cognitive thought.

The next motivation was affective, which included the creative side of the process. The participants created items that were unique and personalized by adding their own ideas, color choices, and stitch preferences in to the overall designs. This was sometimes accomplished by participants who created their own patterns and motifs to be used within 
their knitting. A few were unable to make patterns but still injected their own creative ideas into the projects. Slight alterations in stitches, for example one woman modified the color work pattern on a cap's peak in order to showcase the yarn, allowed the participants to have their own creative influence on the items they produced. Changes in patterns and yarns allowed crafters to have creative control over their projects which increased enjoyment of the process and products (Myzelev, 2009; Turney, 2004). One knitter claimed, “you don’t have to strictly follow a pattern you can sort of make it your own, I think that's part of the creativity of it too rather than just strictly following a pattern" (participant 13). The feeling that patterns could be used as a "roadmap" or source of inspiration was very common amongst the knitters who took the idea of a pattern and then altered it to reflect their own tastes.

The positive reactions and compliments of others on their work established the knitters as accomplished individuals which satisfied their personal integrative needs. The projects made by the knitters were often well received by others. During the interviews many of the knitters described the positive reactions made about their projects. For instance, "I feel great like I make hats and scarves and people always ask me where I got it and I'm like oh I made it” (participant 7). Compliments on finished items often inspired knitters to create more items (Turney, 2004).

Various social integrative needs were fulfilled by knitting and influenced the items produced by the participants. Difficulties encountered while working on their projects were discussed by many of the women. When searching for help a number of sources were popular including other knitters, the Internet, and books or magazines. Some of the knitters had to obtain significant amounts of help of their projects; one 
woman had used help from a local yarn shop to create her project. She explained, "I think I went in there like four different times, I went for like each part of the process” (participant 7). In addition, interaction with other knitters could also produce design ideas. A few knitters described how they might bring a pattern to "Knit Night" and ask for suggestions on yarn choice or even ideas for alterations. Help and input from members of knitting groups has been found by researchers to alter the final projects of knitters (Prigoda \& McKenzie, 2007).

Tension release was identified as one of the main motivators for knitters to engage in knitting. Portability and being able to work on projects in any number of venues was particularly important to the participants. The desire for portability to enable relaxation in numerous situations was one of the main reasons that a majority of projects were accessories. For instance, “I don’t like doing things that are big too much; I don’t like things that are too big to take with me” (participant 8). Researchers have noted that the portability and flexibility of knitting projects enable knitters to take their work to a multitude of locations which allows increased access to benefits from participation, such as relaxation (Rosner \& Ryokai, 2009).

\section{Research Question Number Four}

How do the physical artifacts that are produced by young, female knitters relate to their specific social and political culture? Question four was answered by the material culture analysis, interviews, and the PIPS. The physical items created by the knitters were evident of their specific social and political culture. There were a number of elements from the shared social culture of knitters that influenced the physical artifacts 
created: financial considerations, comfort with digital sources, enjoyment of knitting groups, and involvement with popular culture.

\section{Social Culture}

The cost associated with knitting was as important factor for the participants; eleven (79\%) of the knitters earned under $\$ 25,000$ a year. The financial considerations made by the participants were in opposition to the literature which claimed that knitting is now associated with consumption (Parkins, 2004; Turney, 2009). Surprisingly, the women in the sample had developed ways to be thrifty about their knitting much like the housewives of the past. The participants had discovered a number of ways to reduce the cost of knitting including coupons and sale shopping. The desire to diminish costs can be seen in the physical artifacts which were produced by the knitters. The most popular price range for an artifact was between free and nine dollars. According to the material culture analysis fifteen (51\%) of the artifacts cost under nine dollars to produce. The knitters were able to source materials from thrift stores, using store coupons and some used yarn that had been a gift. The financial consideration were also in the PIPS, eleven (79\%) of the participants spent under $\$ 25$ a month on knitting supplies.

The participants were extremely comfortable with the Internet and most utilized it to forward their knitting. Researchers have found that adoption of technology such as the Internet by crafters is one way for them to enhance their lives as well as the physical artifacts they create (Johnson \& Hawley, 2004). The participants utilized websites like www.ravelry.com to search for patterns and also to share their results with the larger knitting community. The Internet was also used to find instruction on techniques, for example using www.youtube.com to locate a video on how to work a specific stitch. In 
addition, there were many events and groups for the women to participate in. Creating items for swaps and events like Ravolympics influenced the physical items created. As a byproduct of the participation in online groups, the knitters were able to forge important friendships in the virtual world (Rosner \& Ryokai, 2009).

The participants who were involved in various knitting groups also found that their projects were impacted by interactions with other members. Patterns were frequently passed around between knitting friends and also at "Knit Nights." The impact of knitting group members by trading patterns and giving input on the design decisions has been observed by researchers (Prigoda \& McKenzie, 2007). Less skilled knitters reported that they often created items other knitters in their group had already made. For example:

I also do projects sometimes too that I know people have already done so they can give me advice on it or say like oh that part is super easy, but there's this one textual part that's confusing, but I can help you get there. (participant 3)

Researchers have noted a trend of creating items inspired by popular culture; two of these were brought for inclusion in the study (Turney, 2009). A pair of mittens which were inspired by ones worn in the movie Twilight were made by one particiapnt. Additionally, projects with colors from the Harry Potter series were also mentioned by two of the participants. Comfort with popular culture and utilizing it in everyday wear is typical of dress in postmodern society (Morgado, 1996).

\section{Political Culture}

The political culture of the knitters was composed of interest in sustainability, social causes, and group dynamics. The women involved in knitivism showcased an 
interest in social causes as well. The participants reported that it was rewarding for them to use their skills to help other less fortunate individuals. However, knitivism as a whole was less popular with the participants and only three of the women had engaged in any form of this activity. The support of social causes through their knitting was evident of the specific areas of concern to the participants (Pace, 2007; Pentney, 2008).

While the participants were not involved in an environmental knitivism projects, there was an interest in sustainability. The physical products made by the knitters reflected an interest in sustainable fibers and materials. "Green” lifestyles are important to younger consumers, a growing portion of which are generally interested in the health of the environment and make purchase decisions to reflect this awareness (Autio, Heiskanen, \& Heinonen, 2009). The interest in environmental responsibility was seen in the knitters, who fall into the identified age group of consumers most interested in green. Several of the participants discussed using non-dyed, natural animal fibers and others were using eco-friendly fibers. There have been a number of new sustainable yarns available for purchase by knitters (Minihan \& Wolfram Cox, 2007). In the PIPS, seven (50\%) of the women had reported buying eco-friendly yarns in the past year. Another route towards more green knitting employed by the knitters was to use recycled fibers which could be obtained from scrap bins or other projects. Using scrap bin yarns or extra yardage left over from other projects is common for knitters but has not been specifically studied by researchers (Hiatt, 1988; Stoller, 2003).

Group dynamics were another area in which the politics of knitters were demonstrated. Within the knitting groups there was a perceived lack of competitiveness which was enjoyed by the participants. Members were happy to interact with each other 
and share tips and teach techniques. Researchers have supported the claim that experienced members within knitting circles tend to teach and offer advice to less experienced members (Prigoda \& McKenzie, 2007). There appeared to be a teaching hierarchy within the knitting groups to which the participants were members. The teaching hierarchy was explained by one participant, who stated, "it’s definitely kind of a cycle, like they helped you and now you have to pass it on and help the next person” (participant 3). The most experienced members of a knitting group were less likely to teach the newest members. Instead, the beginners were taught by junior members of the group and in turn the junior members were helped by the senior members.

\section{Model}

The following model, The Model of Motivations for Knitting among Young Women, was developed to graphically explain the UG of young female knitters; it is below in Figure 5.1. All of the participants had tension release motivation which is at the base of the model. Knitters in this sample had cognitive, affective, personal integrative and social integrative needs fulfilled by participating in the knitting process which are represented by the four isosceles triangles in the middle of the model. Upon completion of the knitting process, the knitters experienced some form of affirmation, represented by the bar at the top of the model. The feelings of affirmation generally resulted in the knitters' motivation to start a new project, therefore moving from the top of the model to the base to pursue another knitting endeavor. 
Figure 5.1

The Model of Motivations for Knitting among Young Women

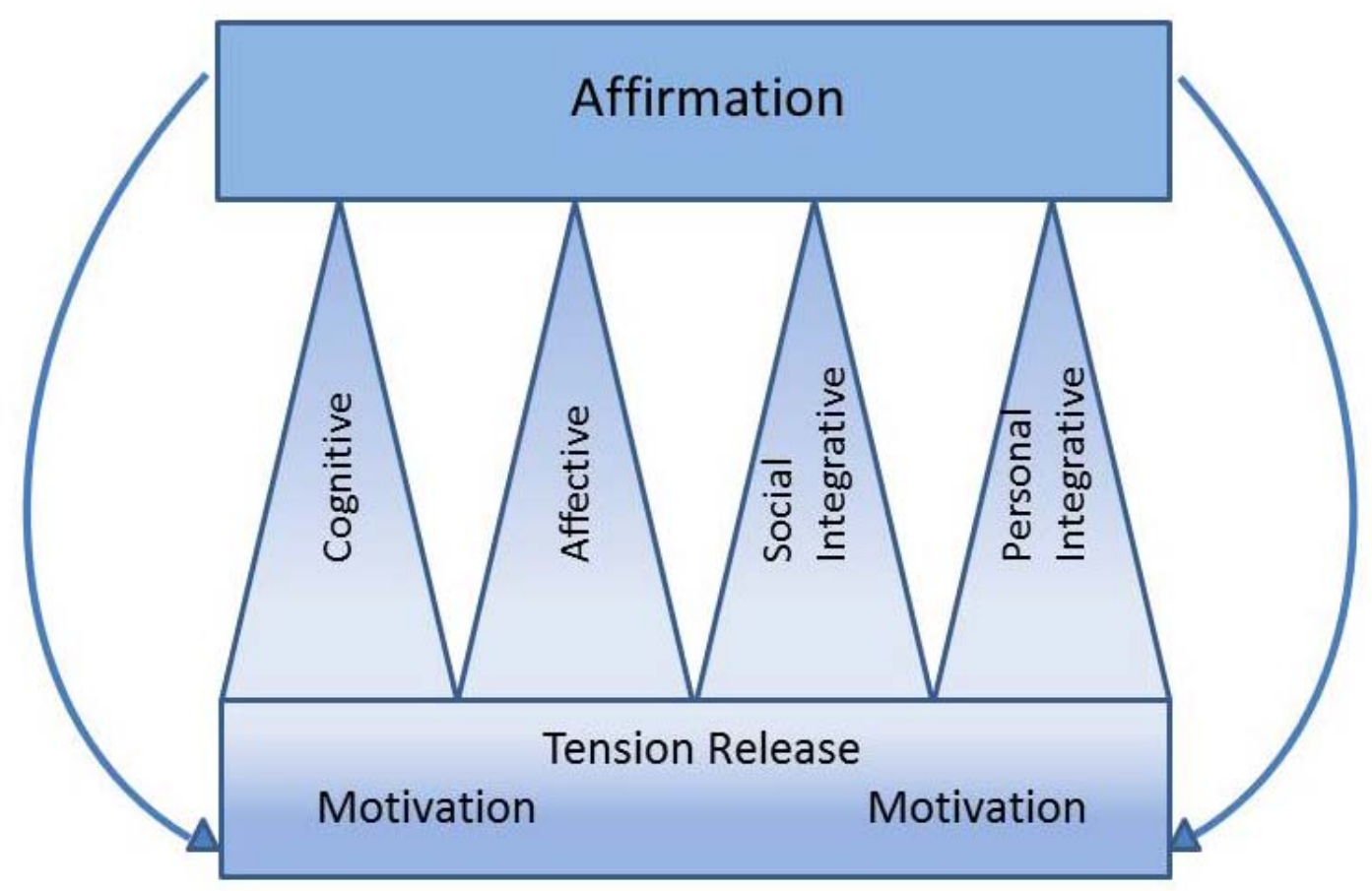

To demonstrate the application of the model, the knitters in this sample, may have had one or a combination of their needs met during the knitting process. For example, one woman used her involvement in knitting groups to fulfill both social integrative and tension release needs. She stated:

You get to know people who you wouldn't meet otherwise, but you have all these similar interests because for all of us its' something that we do just for fun and it's kind of a way to relax and unwind (participant 4).

Another example of a combination of motivations was exhibited by one knitter who used knitting to satisfy her cognitive and affective motivations in addition to tension release. The participant commented, “I'm really inspired by older patterns and sort of the 
history of textiles in general...like really old knitting patterns, and how people figured patterns out and pass them on to each other" (participant 8). The participant discussed history as a source of inspiration which related to creative/affective needs. In addition, the knitter also mentioned how patterns were figured out which exhibits to an interest in cognitive processes.

The various combinations of needs on the participants were met by the knitting process and final projects led to some sort of affirmation. Affirmation came from many sources such as other knitters, the general public, gift recipients, or even from the knitters themselves. Positive affirmations encouraged knitters to continue to participate in knitting. For example, "I like when I get compliments and I can say that I made it" (participant 3). When the knitter received compliments she was able to say she had made the item which satisfied her personal integrative needs and encouraged her to create more items to receive more compliments (affirmations) and further fulfill personal integrative needs.

Thus, knitting was part of a cyclical process, various combinations of motivations were fulfilled which led to affirmation. Endorsement of the final projects, the knitting process, and knitters themselves was extremely important to the participants. In turn affirmation led to more motivations to continue knitting in order to satisfy additional needs. 


\section{CHAPTER SIX}

\section{CONCLUSION}

This research examined the motivations young women have for participating in knitting and the artifacts they produce. A sample of fifteen knitters from the Northern Colorado area was identified for inclusion in the research. Semi-structured interviews, a knitted artifact analysis and post-interview participant surveys, were conducted as part of the research. Data were analyzed using content analysis, material culture methods, and running of frequencies and descriptive statistics. Data from the interviews yielded three major themes: incentives, barriers, and outcomes and thirteen sub-themes. The major and sub-themes were outlined in the Taxonomy of Participation in Knitting, which was developed to visually explain the aspects of knitting as experienced by young, female knitters. The results were also compared to an existing theory from mass media studies, Uses and Gratifications (UG).

The theme of incentives delved into the various motivations and supports available to knitters. The theme examined the role of social aspects in the culture of current knitters and the multitude of accessible information sources. Advantages of knitting including creativity and the ability to knit simultaneously with other activities were also highlighted. 
Barriers to knitting examined the negative aspects of the process and their effect on the desire of knitters to craft. Knitters encountered a number of negative reactions such as teasing and over simplification of their hobby. Mistakes on the part of the knitter also caused harmful responses and sometimes self-criticism and doubt.

The outcomes from the knitting process helped to bolster the confidence of the knitters and encouraged continued involvement. The resulting products were well regarded and fostered a sense of uniqueness and even a feeling that the individual could become self-sustained through their knitting. One of the most significant results of the knitting process was a sense of relaxation which was encouraged the knitters to continue to participate.

Uses and Gratifications was compared to the data to determine if there was a correlation between the categories defined by Katz et al. (1974) and the motivations the participants had for knitting. The categories were very appropriate for describing the knitters and as a result of the research The Model of Motivations for Knitting among Young Women was developed to graphically explain the relationship between motivations and benefits from engaging in knitting. The categories of motivations that knitting satisfied were cognitive, affective, personal integrative, social integrative, and tension release. Interestingly, all of the knitters had tension release motivations.

\section{Significance}

This study is significant as the documentation of knitters is sparse. Young knitters and their material culture, in particular, had never been studied. By looking at knitting from a non-guild standpoint it was possible to document the wider trend by examining knitters from differing backgrounds, levels of participation, and social 
standpoints. The knitted artifacts produced by the participants were documented in an effort to not only understand the motivations and benefits of knitting, but also some of the design decisions made by knitters.

The Taxonomy of Participation in Knitting graphically explains the aspects of participation in knitting for young women. All of the participants had tension release motivations to participate in knitting. Additional motivations for engaging in knitting included: cognitive, affective, personal integrative and social integrative. A resulting model, The Model of Motivations for Knitting among Young Women, shows how the incentives and benefits of knitting are interrelated and how they related to the motivations of participants.

\section{Implications}

This research will impact the young women who have been active in the resurgence and redefinition of knitting as a trendy pursuit. It is the first study to examine the specific material culture artifacts created by these women and to recognize the skill, effort, and time involved in hand knitted items. By highlighting and appreciating the efforts of young knitters it is possible to elevate their activities beyond simple craft.

The findings of this research are of interest to craft retailers and authors of knitting publications. Preferences for yarns, fibers, stitches, and types of projects were discussed in depth. It would be valuable to retailers and authors to understand the requirements and inclinations for knitting projects of young, female knitters.

This study will also impact academic researchers by exploring the subject and creating research for future works to compare against. Both of the models, which resulted from this research will be useful to further studies on the motives of crafters. As 
part of the slowly growing body of research focused on traditional textile handcrafts, this study will help to bring further attention and understanding to the important of crafts.

\section{Future Research}

There are several areas in which future research should be conducted. The collection of qualitative data is specific to the population which was studied, so it cannot be applied to all young knitters. The sample used for this study all presently live in the Northern region of Colorado, which could have a significant impact on the products, motives, and social culture of the knitters and reduce the generalizability of the results. Future research should utilize a larger and more geographically diverse sample.

Research on traditional textile handcrafts tends to be descriptive in nature; future studies should emphasize the use of theory. For example, the application of Uses and Gratifications to this research was successful and allowed for the creation of a model. Uses and Gratifications should continue to be used to explain the motivations of individuals for participating in other traditional textile handcrafts.

Due to the way comparable data were gathered from www.ravelry.com it was not possible to fully understand some of the provenances and values of the artifacts. Comparable data should be collected from blogs so that the artifacts will be more completely explained and easier to compare to the sample artifacts. Comparable data could also be gathered from different population of knitters such as older women to generate a comparison between different knitters’ material cultures.

A contrast between knitting and other traditional textile handcrafts could prove to be quite valuable. Quilting for example has enjoyed the same renewed attention as knitting and it would be interesting to compare between both crafts. A comparison 
between a traditional craft that has not undergone a revival such as silk ribbon embroidery or tatting could also provide valuable information as to what aspects of crafts result in a social trend.

The dependence of young female knitters on websites such as www.ravelry.com demonstrates the importance of these sites in directing trends among knitters. An analysis of these websites would prove helpful in detailing the overall material culture of knitters. Furthermore, it would be worthwhile to inspect the discussions held online by knitters to examine the design decisions and contributions of members. 


\section{REFERENCES}

Abrams, L. (2006). Knitting, autonomy and identity: The role of hand-knitting in the construction of women's sense of self in an island community, Shetland, c. 18502000. Textile History, 37(2), 149-165.

Arai, S., \& Pedlar, A. (2003). Moving beyond individualism in leisure theory: A critical analysis of concepts of community and social engagement. Leisure Studies, 22(1), 185-202.

Arneil, B. (1999). Politics and feminism. Malden, MA: Blackwell Publishers Ltd.

Autio, M., Heiskanen, E., \& Heinonen, V. (2009). Narratives of 'green’ consumers: The antihero, the environmental hero, and the anarchist. Journal of Consumer Behavior, 8(1), 40-53.

Belcastro, S. \& Yackel, C. (Eds.). (2008). Making mathematics with needlework. Wellesley, MA: A K Peters, Ltd.

Blood, J. A. (2006). Non-industrial textile production as optimal experience: Applicability of the flow theory to clothing and textiles subject matter. (Unpublished doctoral dissertation). Oregon State University, Oregon.

Campbell, C. (2005). The craft consumer: Culture, craft and consumption in a postmodern society. Journal of Consumer Culture, 5(1), 23-42.

Chansky, R. (2010). A stitch in time: Third-wave feminist reclamation of needled imagery. The Journal of Popular Culture, 43(4), 681-700.

Cho, Y. C. (2007). Measuring customer attitudes toward single vs. hybrid retail formats: Impact of gender and brand name familiarity. Journal of Applied Business Research, 23(4), 79-92.

Choi, W., \& Powell, N. B. (2005). Three dimensional seamless garment knitting on v-bed flat knitting machines. Journal of Textile and Apparel Technology and Management, 4(3), 2-33. 
Colton (Ed). (1979). Reader's digest complete guide to needlework. Pleasantville, NY: The Reader's Digest Association, Inc.

Creswell, J. W. (2009). Research design: Qualitative, quantitative, and mixed methods approaches ( $3^{\text {rd }}$ ed. Rev.). Thousand Oaks, CA: Sage Publications Inc.

Csikszentmihalyi, M. (1990). Flow: The psychology of optimal experience. New York, NY: Penguin Books.

Denzin, N. K. \& Lincoln, Y. S. (Eds.). (2000). Handbook of qualitative research ( $2^{\text {nd }}$ ed. Rev.). Thousand Oaks, CA: Sage Publications Inc.

Donofrio-Ferrezza, L. \& Hefferen, M. (2008). Designing a knitwear collection. New York, NY: Fairchild Books Inc.

Edwards, D. C. (1999). Motivation and emotion: Evolutionary, physiological, cognitive, and social influences. Thousand Oaks, CA: Sage.

Eig, E. (2002, Jan). Yen for yarn. People, 57(2), 71.

Eighmey, J. \& McCord, L. (1998). Adding value in the information age: Uses and gratifications of sites on the World Wide Web. Journal of Business Research, 41(1), 187-194.

Engel-Enright, C. (2007). The needs, desires, activities, and skills of independent clothing designers as small business owners. (Unpublished master's thesis). Colorado State University, Colorado.

Fleming, E. M. (1982). Artifact study: A model. In Thomas J. Schlereth (Ed.), Material culture studies in America (p. 162-163). Nashville, TN: The American Association for State and Local History.

Gamble, S. (Ed.). (1999). The Routledge critical dictionary of feminism and postfeminism. New York, NY: Routledge.

Gibson, W. J. \& Brown, A. (2009). Working with qualitative data. Thousand Oaks, CA: Sage.

Haitt, J. H. (1988). The principles of knitting. New York, NY: Simon and Schuster.

Harris, J.(Ed.). (2004). 5,000 years of textiles. Washington, DC: Smithsonian Books.

Harris, M. (1988). Common threads: Mathematics and textiles. Mathematics in School, 17(4), 24-28. 
Hawley, J. M. (2005). The commercialization of Old Order Amish quilts: Enduring and changing cultural meanings. Clothing and Textiles Research Journal, 23(2), 102114.

Hollingworth, S. (1982). The complete book of traditional Aran knitting. London, UK: B. T. Batsford, Ltd.

Hollows, J. (2003). Feeling like a domestic goddess: Postfeminism and cooking. European Journal of Cultural Studies, 6(2), 179-202.

Hunt, K. (2005). Pearls of Wisdom: Motivations of contemporary women knitters. (Unpublished master's thesis). Iowa State University, Iowa.

Johnson, S. J., \& Wilson, L. E. (2005). “It says you really care”: Motivational factors of contemporary female handcrafters. Clothing and Textiles Research Journal, 23(2), 115-130.

Kaiser, S. B. (1997). Social psychology of clothing: Symbolic appearances in context $\left(2^{\text {nd }}\right.$ ed. Rev.). New York, NY: Fairchild.

Katz, E., Blulmer, J. G., \& Gurevitch, M. (1974). Utilization of mass communication by the individual. In Jay G. Blumler and Elihu Katz (Eds.), The uses of mass communications: Current perspectives on gratifications research (p. 19-34). Beverly Hills, CA: Sage publications LTD.

Ko, H., Cho, C., \& Roberts, M. S. (2005). Internet uses and gratifications. Journal of Advertising, 34(2), 57-70.

Macdonald, A. L. (1988). No idle hands: The social history of American knitting. New York, NY: Ballantine Books.

McQuail, D., Blumler, J. G., \& Brown, J. R. (1972). The television audience: A revised perspective. In D. McQuail (ed.), Sociology of Mass Communications, 135-165. Harmondsworth, Eng.: Penguin.

Medford, K. M. (2006). I knit therefore I am: An ethnomethodological study of knitting as constitutive of gendered identity. (Unpublished doctoral dissertation). Bowling Green State University, Ohio.

Minahan, S., \& Wolfram Cox, J. W. (2007). Stitch ' $n$ Bitch: Cyberfeminism, a third place and the new materiality. Journal of Material Culture, 12(1), 5-21.

Mondi, M., Woods, P., \& Rafi, A. (2008). A uses and gratifications expectancy model to predict students perceived e-learning experience. Educational Technology \& Society, 11(2), 241-261. 
Morgado, M. A. (1996). Coming to terms with postmodernism: Theories and concepts of contemporary culture and their implications for apparel scholars. Clothing and Textile Research Journal, 14(1), 41-53.

Myzelev, A. (2009). Whip your hobby into shape: Knitting, feminism and construction of gender. Textile, 7(2), 148-163.

No Author. (2010). Exotic yarns. Retrieved from http://www.yarn.com/webs -knitting-crochet-yarns-fiber-exotic/.

Ogle, J., Hyllegard, K., \& Dunbar, B. (2004). Predicting patronage behaviors in a sustainable retail environment: Adding retail characteristics and consumer lifestyle orientation to the belief-attitude-behavior intention model. Environment and Behavior, 36(5), 717-741.

Pace, L. A. (2007). Changing the world one stitch at a time: Knitting as a means of social and political action. (Unpublished master's thesis). University of Akron, Ohio.

Parkins, W. (2004). Celebrity knitting and the temporality of postmodernity. Fashion Theory, 8(4), 425-442.

Pentney, B. A. (2008). Feminism, activism, and knitting: Are the fibre arts a viable mode for feminist political action. Third Space: A Journal of Feminist Theory and Culture, 8(1), Retrieved February 1, 2010, from http://www.thirdspace.ca/journal/article/view/pentney/210

Prigoda, E., \& McKenzie, P. J. (2007). Purls of wisdom: A collectivist study of human information behavior in a public library knitting group. Journal of Documentation, 63(1), 90-114.

Prown, J. D. (1982). Mind in matter: An introduction to material culture theory and method. Winterthur Portfolio, 17(1), 1-19.

Prown, J. D. (2000). Preface. In J. D. Prown, \& K. Haltman (Eds.), American artifacts: Essays in material culture (p. ix-xiii). East Lansing, MI: Michigan State University Press.

Reynolds, F. (1997). Coping with chronic illness and disability through creative needlecraft. British Journal of Occupational Therapy, 60(8), 352-356.

Reynolds, F. (2009). Taking up arts and crafts in later life: A qualitative study of the experiential factors that encourage participation in creative activities. British Journal of Occupational Therapy, 72(9), 393-400. 
Rosner, D. K., \& Ryokai, K. (2009, October). Reflections on craft: Probing the creative process of everyday knitters. Paper presented at the meeting of the Seventh ACM Conference on Creativity and Cognition, Berkeley, CA.

Rutt, R. (1987). A history of hand knitting. Loveland, CO: Interweave Press.

Schofield-Tomschin, S., \& Littrell, M. A. (2001). Textile handcraft guild participation: A conduit to successful aging. Clothing and Textiles Research Journal, 19(2), 41-51.

Searly, K. (2008). Knitting art: 150 innovative works from 18 contemporary artists. Minneapolis, MN: Voyageur Press.

Severin, W. J., \& Tankard, J. W., Jr., (Eds.). (1997). Communication theories: Origins, methods, and uses in the mass media ( $4^{\text {th }}$ ed.). White Plains, NY: Longman Publishers.

Skinitis, A. (October 10, 2009). The moment: Rent a grannie. The Times. Retrieved: http://women.timesonline.co.uk/tol/life_and_style /women/fashion/article6861622.ece.

Smith, S. (1985). Towards a material history methodology. Material History Bulletin, 22(1), 31-40.

Springgay, S. (2010). Knitting as an aesthetic of civic engagement: Re-conceptualizing feminist pedagogy through touch. Feminist Teacher, 20(2), 111-123.

Stanfield, L. (2007). 150 knitted trims. New York, NY: St. Martin’s griffin.

Stein, B. D., Elliot, M. N., Jaycox, L. H., Collins, R. L., Berry, S. H., Klein, D. J., \& Schuster, M. A. (2004). A national longitudinal study of the psychological consequences of the September 11, 2001 terrorist attacks: Reactions, impairment, and help-seeking. Psychiatry, 67(2), 105-117.

Stoller, D. (2003). Stitch 'n bitch: A knitter’s handbook. New York, NY: Workman Publishing.

Strawn, S. M. (2007). Knitting America: A glorious history from warm socks to high art. St. Paul, MN: Voyager Press.

Strauss, A., \& Corbin, J. (1990). Basics of qualitative research: Grounded theory procedures and techniques. Newbury Park, CA: Sage.

Tashakkori, A. \& Teddlie, C. (1998). Mixed methodology: Combining qualitative and quantitative approaches. Thousand Oaks, CA: Sage. 
Turney, J. (2004). Here's one I made earlier: Making and living with home craft in contemporary Britain. Journal of Design History, 17(3), 267-281.

Turney, J. (2009). The culture of knitting. New York, NY: Berg Publishers.

Walker, B. G. (1971). The craft of lace knitting. New York, NY: Charles Scribner’s Sons.

Wayment, H. A. (2006). Attachment style, empathy, and helping following a collective loss: Evidence from the September 11 terrorist attacks. Attachment \& Human Development, 8(1), 1-9.

Zawilinski, K. (2010, May). Ten eco-friendly yarns you'll love. Better Homes and Gardens. Retrieved from http://www.bhg.com/crafts/knitting/basics/eco-friendlyyarns-youll-love. 
APPENDIX A: Examples of Knitivism 


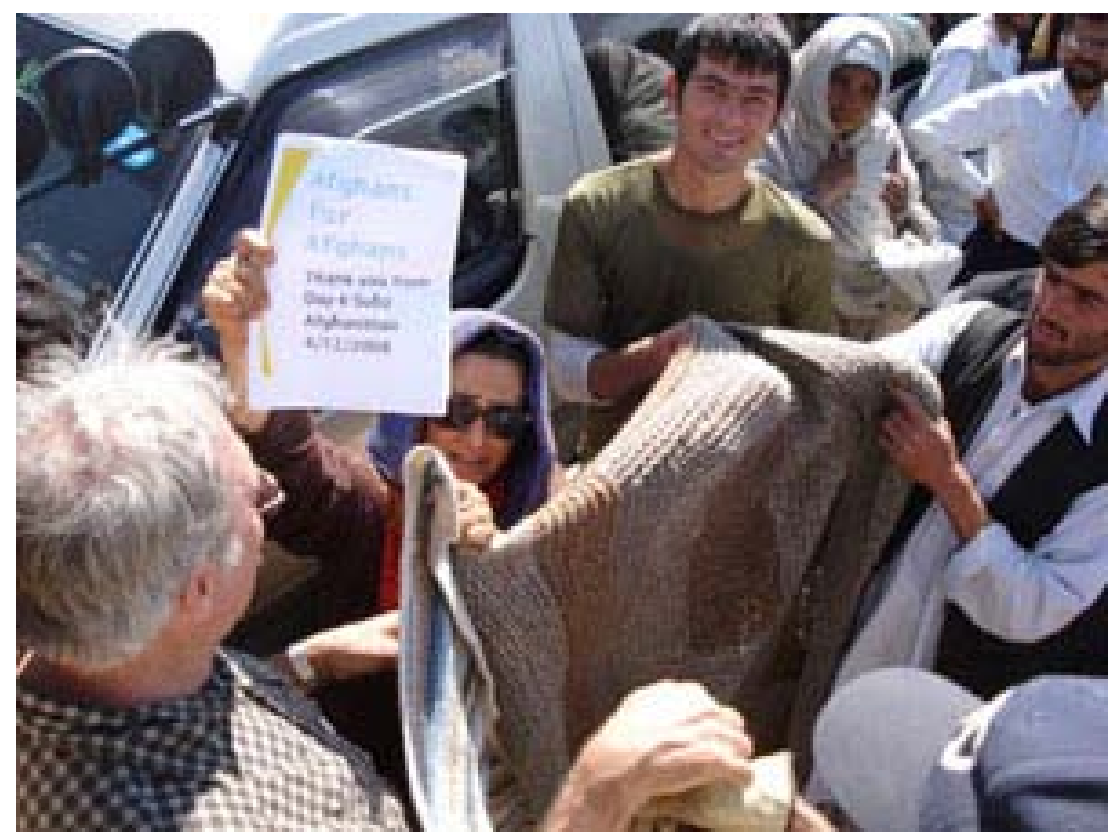

Afghan delivery day in Afghanistan . Available from http:// www.afghansforafghans.org.

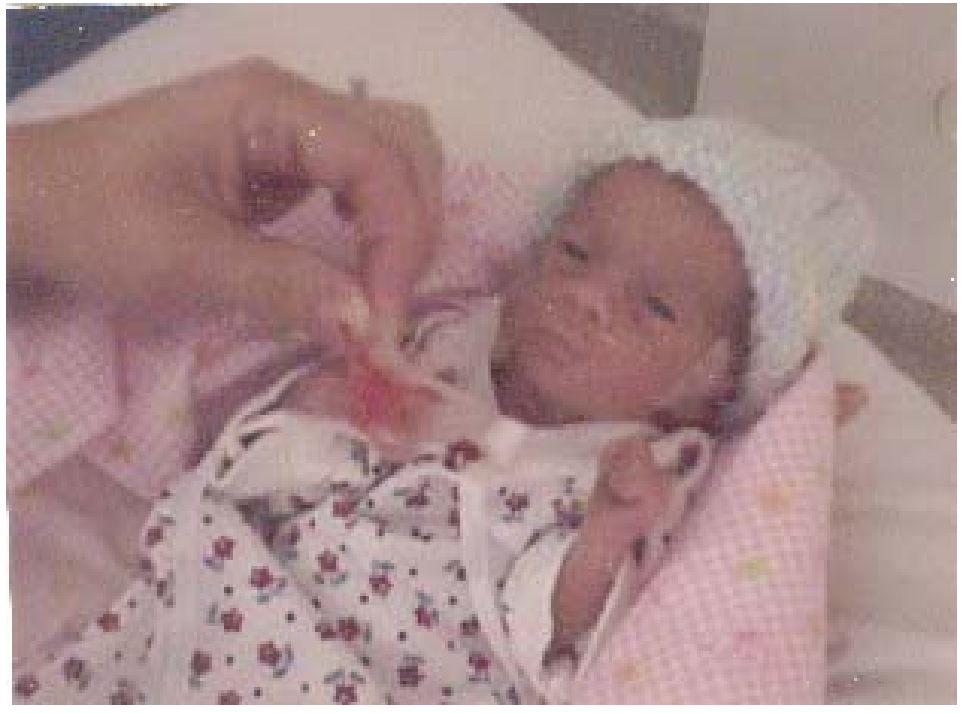

A knitted cap for premature babies (Pace, 2007, p. 13) 


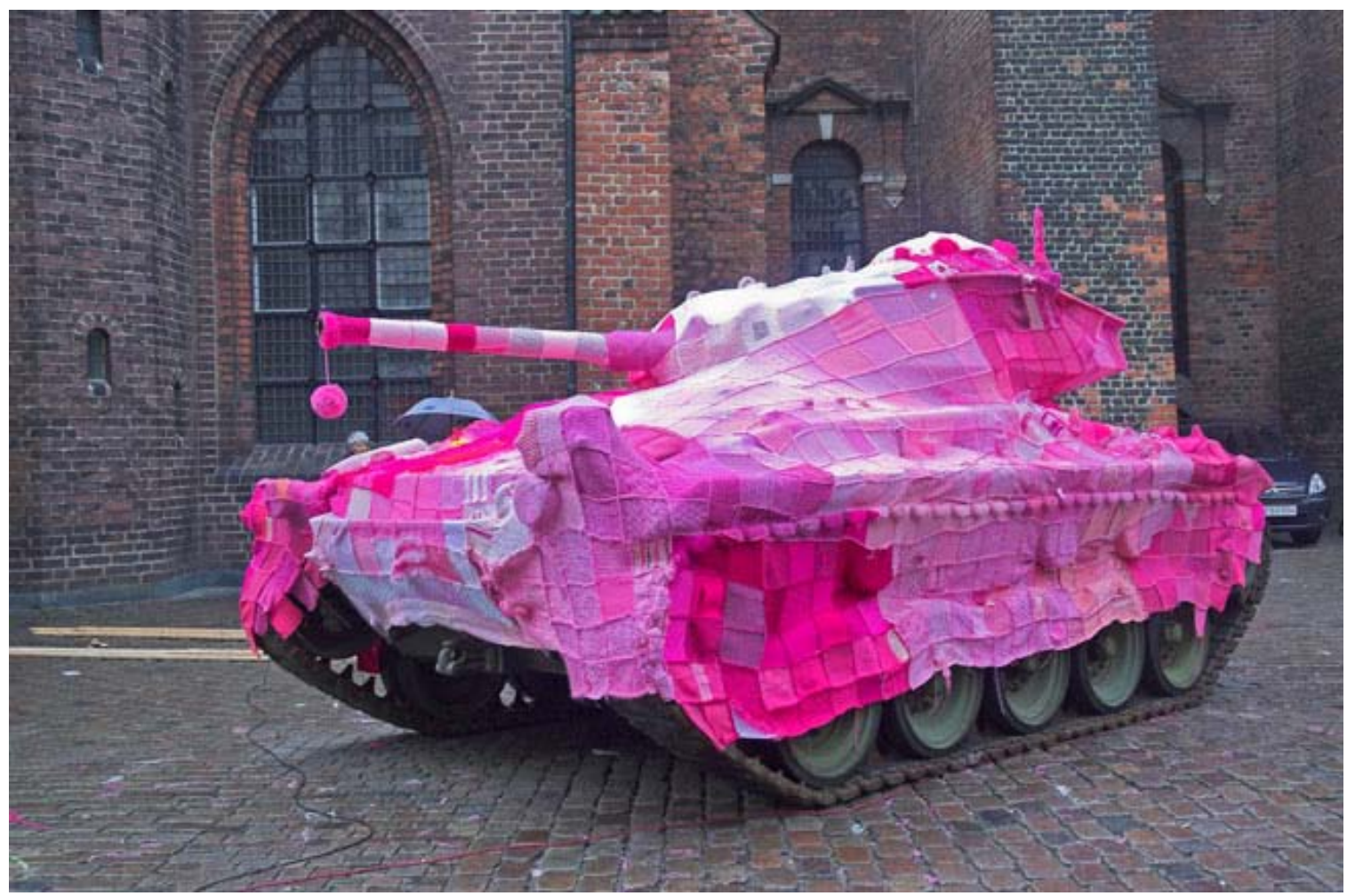

A knitted tank cover by artist Marianne Jorgensen. Retrieved from: http://www.marianneart.dk/

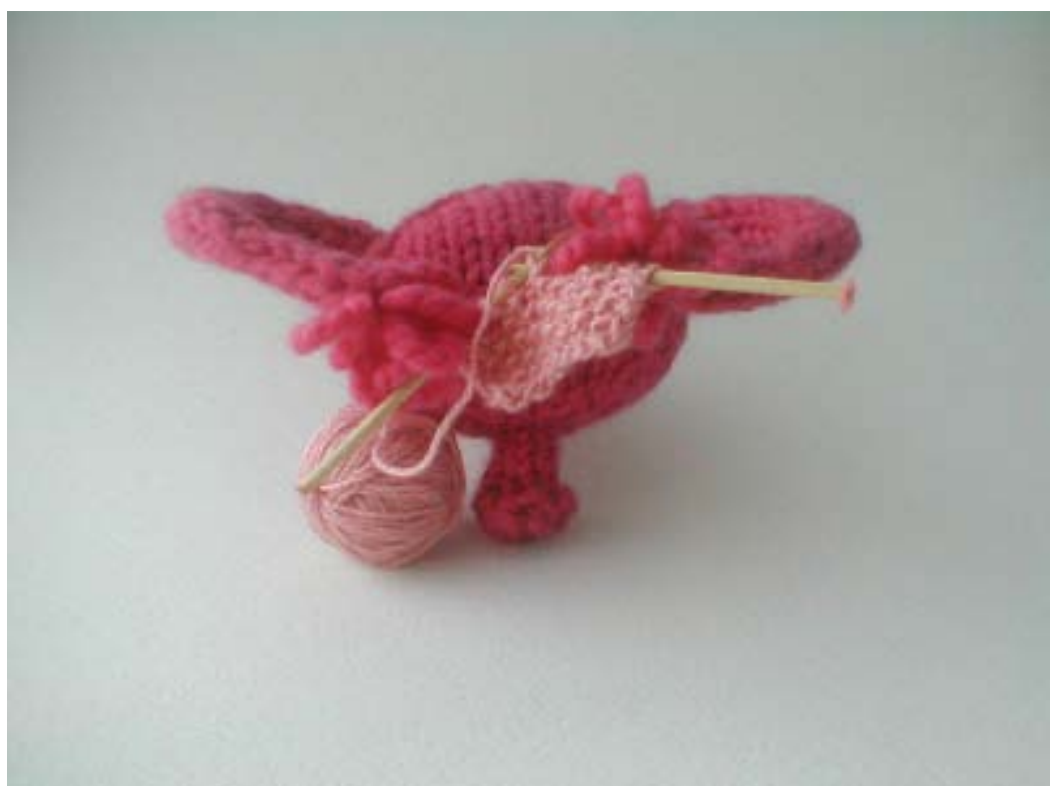

A knitted womb from the Wombs on Washington Project (Pentney, 2008) 


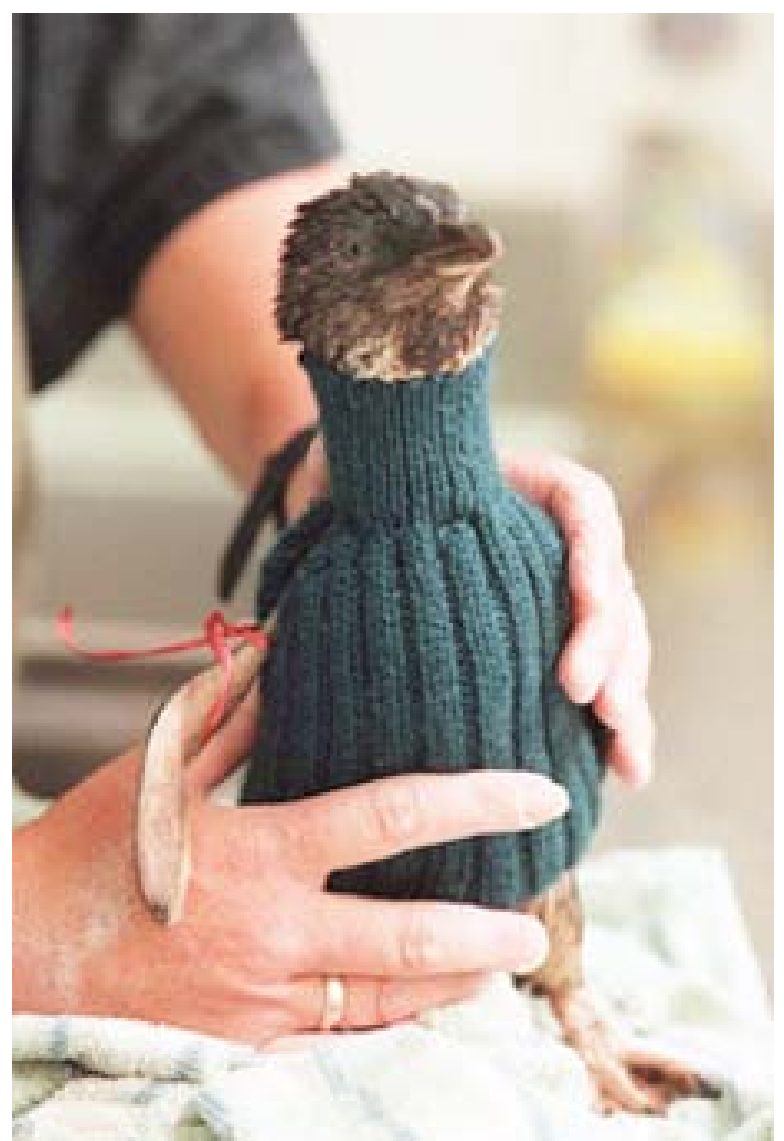

A knitted sweater to help fairy penguins after an oil spill (Pace, 2007, p. 132) 
APPENDIX B: Interview Schedule 


\section{Interview Schedule}

\section{Questions Related to the Artifact}

- What types of decisions (for example yarn type, size, stitches to use, ect.) did you have to make about this project before starting it?

- Why did you pick this yarn and this pattern?

- Did you seek help for this project? If so where? From whom?

\section{Relationship between the Artifact and Knitter.}

- How do you feel when you wear/ use this object?

- Was that the intended outcome of the item?

- Does it suit its original purpose (ex, does it fit or look good)?

- Describe people's reactions to your project.

- How do these make you feel about your abilities and project?

\section{Motivations for Participation in Knitting}

1. How did you learn to knit?

- Who taught you?

- What was the first project you worked on?

- Was there a specific reason you decided to learn?

- Have you taught anyone else?

2. Have you ever had to alter a pattern to fit correctly or changed the motifs within the design?

- Did you find this to be very challenging? Was it fun?

- Do you find that you use math and counting in the knitting process?

3. Do you ever use your own creative ideas for your projects? If so when?

- Where do you tend to find ideas and patterns for your projects?

- Do you have a specific publication, blog, website you visit for knitting information?

4. Do you ever give your projects as gifts?

- If so can you give me an example?

- How did this make you feel?

- Do you have a blog or participate in knitting contests?

- How does the interest of others make you feel? 
- Has your knitting ever been devalued? If so when?

- How did this affect your desire to knit?

5. Are you involved in a knitting community, such as an online group, guild, or knitting circle?

- What is your favorite thing about the group?

6. How does knitting make you feel?

- Does it relieve stress?

- Where and when do you tend to work on your knitting?

7. Do you have anything else you would like me to know? 
APPENDIX C: Knitted Artifact Instrument 


\section{Knitted Artifact Instrument}

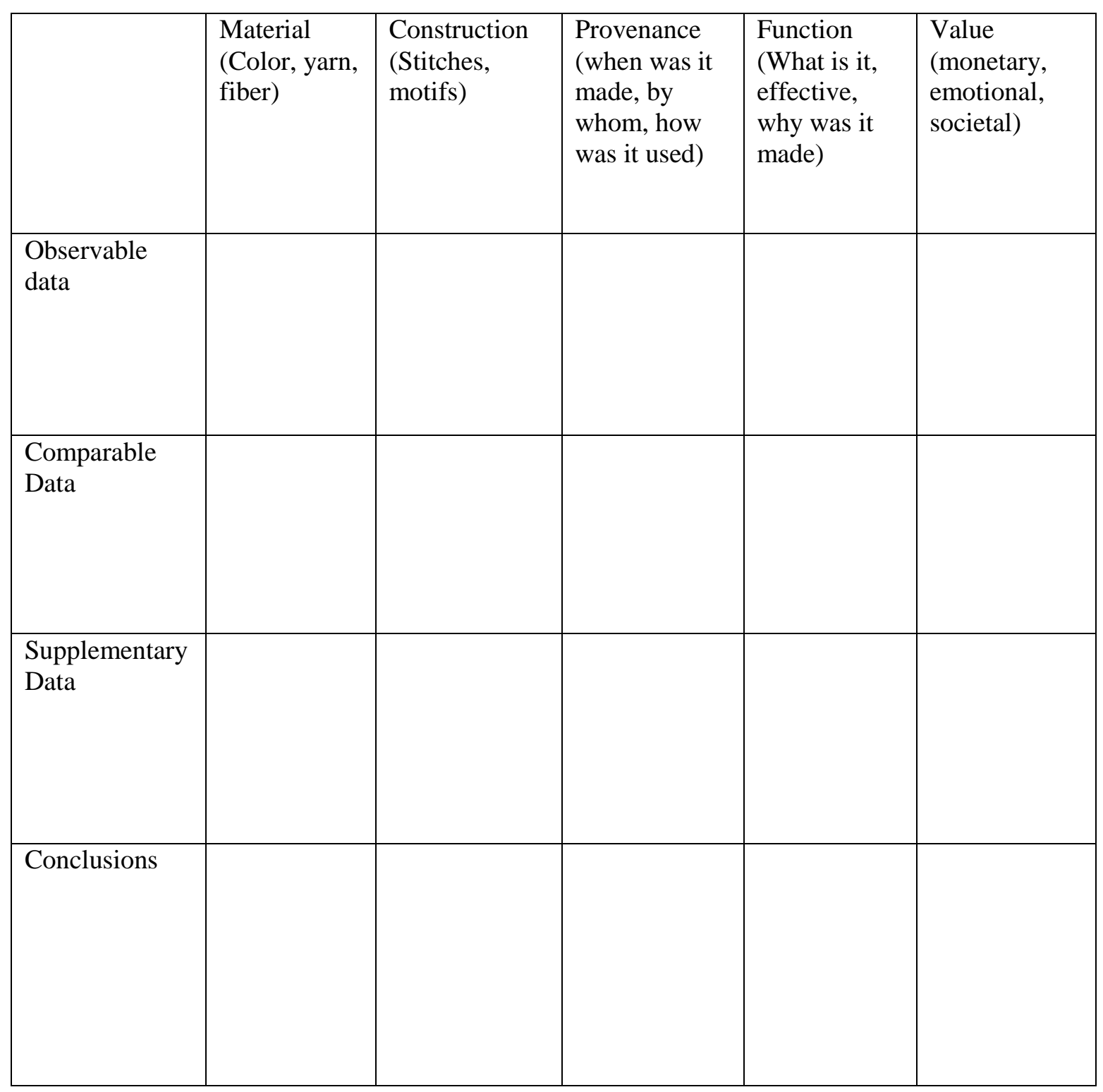


APPENDIX D: Participants for Research Flyer 


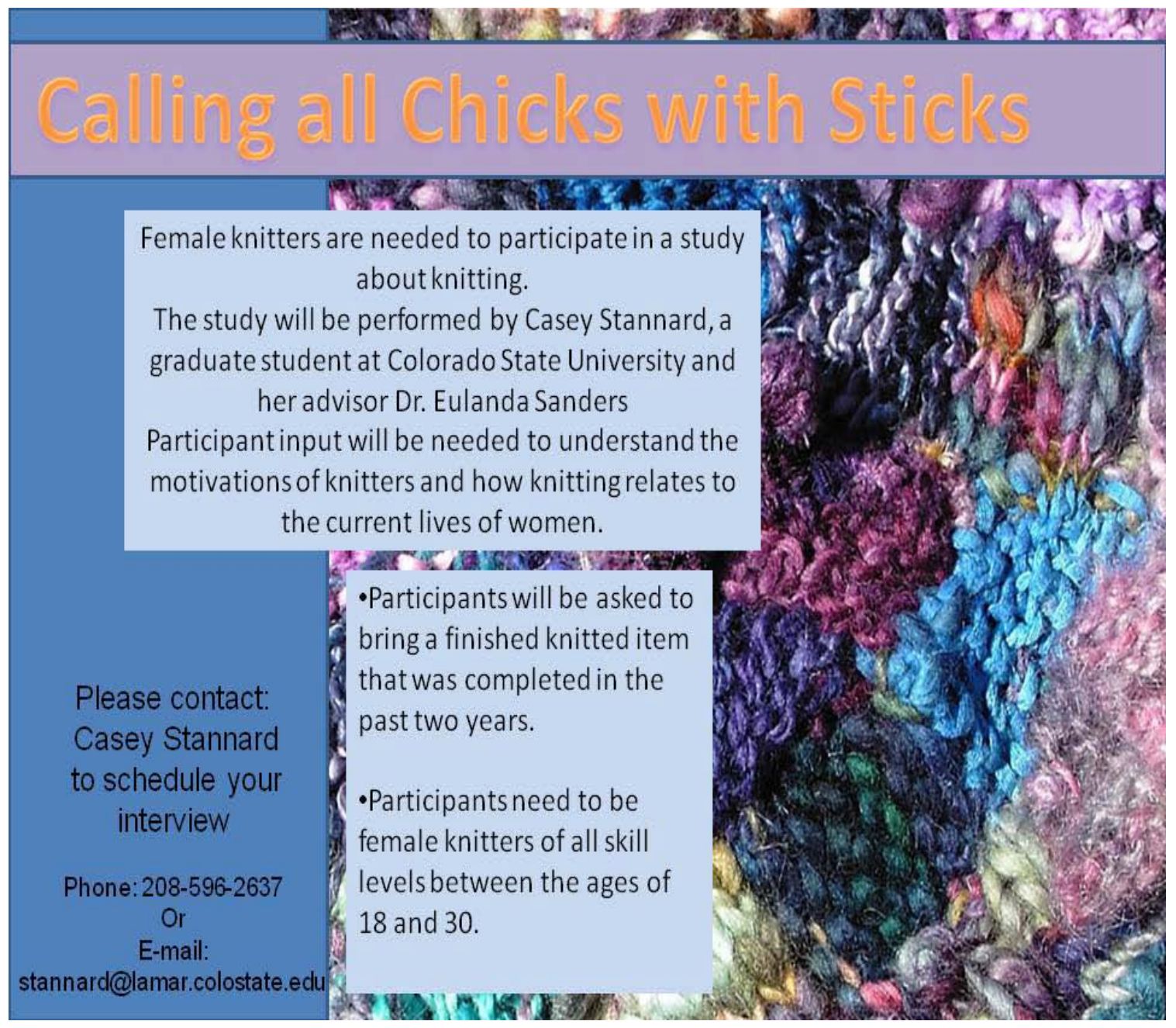


APPENDIX E: Post Interview Participant Survey 


\section{Post-Interview Participant Survey}

Motivations for participation in Knitting among Young Women

Please take a few minutes to fill out this survey on some basic demographic and knitting information. The principle and co-investigators want to assure you that your answers will be kept confidential. Again, thank you for your participation.

\section{General Participant Information}

What is your current age?

What is your highest level of education?

$\begin{array}{cccccc}0 & 0 & 0 & 0 & 0 & \bigcirc \\ \text { High } & \text { Some } & \text { Bachelor's } & \text { Master's } & \text { Doctoral } & \text { Prefer not } \\ \text { school } & \text { college } & \text { degree } & \text { degree } & \text { Degree } & \text { to answer }\end{array}$

What is your level of yearly income?

$\begin{array}{cccccc}0 & \bigcirc & 0 & \bigcirc & \bigcirc & \bigcirc \\ <\$ 10,000 & \$ 10,000- & \$ 25,000- & \$ 40,000- & \$ 65,000- & >\$ 80,000 \\ & \$ 25,000 & \$ 40,000 & \$ 65,000 & \$ 80,000 & \end{array}$

\section{Knitting information}

How many years have you been knitting?

Do you consider yourself to be a process knitter (someone who enjoys the act of knitting more than the final product) or do you consider yourself to be a product knitter (someone who enjoys the final product more than the act of knitting)?

○

Process

Knitter
Product

Knitter

How much time do you spend knitting in an average week?

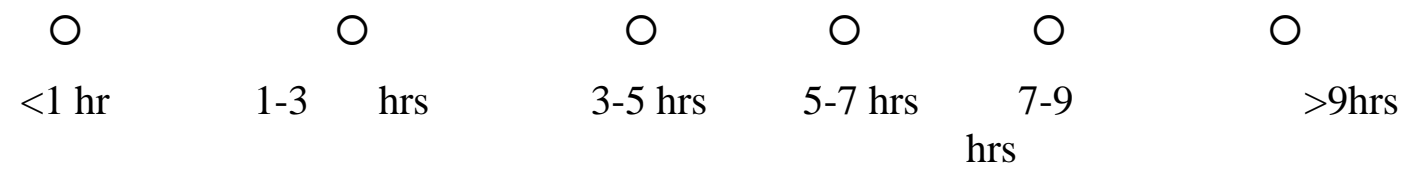


How much time do you spend on knitting supplies in an average month?

$\begin{array}{cccccc}0 & 0 & 0 & 0 & 0 & 0 \\ <\$ 10 & \$ 10-\$ 25 & \$ 25-\$ 40 & \$ 40-\$ 65 & \$ 65-\$ 80 & >\$ 80\end{array}$

What types of knitted items have you made in the past year (check all that apply)?

$\begin{array}{lclc}\text { Sweaters } & \bigcirc & \text { Hats } & \bigcirc \\ \text { Other garments } & \bigcirc & \text { Fingerless Gloves } & \bigcirc \\ \text { Gifts } & \bigcirc & \text { Blankets } & \bigcirc \\ \text { Toys or other novelty } & \bigcirc & \begin{array}{l}\text { Other gloves, } \\ \text { mittens }\end{array} & \bigcirc \\ \text { items } & & \end{array}$

What types of yarns have you purchased in the past year (check all that apply)?

$\begin{array}{llll}\text { Acrylic } & \bigcirc & \text { Alpaca } & \bigcirc \\ \text { Cotton } & \bigcirc & \begin{array}{l}\text { Novelty } \\ \text { blends }\end{array} & \bigcirc \\ \text { Silk } & \bigcirc & \text { Hand spun } & \bigcirc \\ \text { Wool } & \bigcirc & \text { Hand dyed } & \bigcirc \\ \text { Mohair } & \bigcirc & \text { Eco-friendly } & \bigcirc\end{array}$

Which knitting techniques do you prefer (check all that apply)?

Cables $\quad 0$ Other

Color Work $\quad 0$

Lace $\quad 0$

Knitting in the round $\mathrm{O}$

Flat knit with seaming $\bigcirc$

How would you rate your level of skill as a knitter?

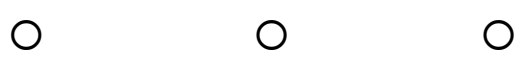

Advanced Intermediate Beginner 


\section{Additional Feedback}

Please share any additional comments.

Thank you for taking the time to fill out our survey. We appreciate you serving as a participant in this research. Your input is greatly appreciated. 